\title{
Effects of additional food in a susceptible-exposed-infected prey-predator model
}

\author{
Banshidhar Sahoo $^{1} \cdot$ Swarup Poria ${ }^{1}$
}

Received: 1 August 2016/Accepted: 13 August 2016/Published online: 26 August 2016

(C) Springer International Publishing Switzerland 2016

\begin{abstract}
In this paper, we propose a susceptible-exposedinfected prey-predator model supplying additional food to predator. We discuss the existence and the local stability conditions of both the disease free and endemic equilibrium points. Basic reproduction number is determined. The impact of additional food on infected prey population is discussed. Numerical simulation results establish that there exists a critical infection rate above which disease present in the system in absence of additional food. However, in such case supply of suitable additional food can make the system disease free. We compute the disease free regions in various parametric planes. Effects of variation of latent period is presented. Season dependent infection rate is also introduce in the system and the role of additional food is discussed. The main goal of this study is to show the nontrivial consequences of providing additional food for controlling infection in a diseased predator-prey system. This study is aimed to introduce a new non-chemical method for controlling disease of prey population.
\end{abstract}

Keywords Infected-prey · Additional food - Basic reproduction number $\cdot$ Local stability $\cdot$ Disease free . Bifurcation

Banshidhar Sahoo

banshivu@gmail.com

Swarup Poria

swarup_p@yahoo.com

1 Department of Applied Mathematics, University of Calcutta, Kolkata, West Bengal, India

\section{Introduction}

Eco-epidemiology is a new branch of study in mathematical biology which incorporates both the ecological and epidemiological issues simultaneously. An eco-epidemiological model is a combination of an ecological model and an epidemic model which may be a SI, SIS or SIR type. In recent years significant progress has occurred in the theory and applications of epidemiology models in predator-prey systems (Kooi et al. 2013; Venturino 2010; Wei et al. 2009; Kermack and McKendrick 1927). These mathematical models help to identify key parameters which determine the rich dynamics of an epidemiological system. Dynamics of transmissible disease in an ecological situation is remaining a major field of study due to its applications in real life. An epidemic model includes the specific property of population growth, the spread rules of infectious diseases, and the related ecological factors to construct mathematical models reflecting the dynamic properties of infectious diseases. The first mathematical description of contagious diseases has been formulated by Kermack and McKendric (1927). They had reported the influence of mathematical models in disease spreading and their models are still relevant in many epidemic situations (Kermack and McKendrick 1932, 1933). Anderson and May (1986) were considered the disease factor in a predator-prey dynamics and found that the pathogen tends to destabilize the predator-prey interaction. In Rosenzweig prey-predator model, an epidemic threshold (above which an infected equilibrium or an infected periodic solution appear) was determined by Hadeler and Freedman (1989). Chattopadhyay and Arino (1999) considered a three species eco-epidemiological model and determined extinction criteria of species and found condition for Hopf-bifurcation in an equivalent two-dimensional model. Haque and 
Chattopadhyay (2007) investigated the role of transmissible diseases in a prey dependent predator-prey system with prey infection.

Most of the literature of epidemic models assume that the disease incubation is negligible so that, once species becomes infected, each susceptible individual $(S)$ instantaneously becomes infectious (I) and later recovers (R) with a permanent or temporary acquired immunity. A compartmental model based on these assumptions is called a SIR or SIRS model. However, many diseases incubate inside the hosts for a period of time before the hosts become infectious. In other words a susceptible individual first goes through a latent period (and is said to become exposed or in the class E) after infection before becoming infectious. The resulting models are of SEI, SEIR or SEIRS type, respectively, depending on whether the acquired immunity is permanent or not. A susceptible individual (S) in contact with an infected individual either becomes exposed (E) or becomes infected (I) individual. Exposed and infected individuals remain forever in the same state. Susceptible individual becomes exposed or infected with rates that are proportional to the number of neighbouring infected individuals. The first reaction $(S \rightarrow E)$ is catalytic whereas the second $(S \rightarrow I)$ is auto catalytic reaction (Tomé and de Oliveira 2011).

The basic reproduction number $R_{0}$ is the most important quantity in the study of epidemics model. Epidemic models admit only a globally asymptotically stable disease free equilibrium if $R_{0}<1$ (Zhou and Cui 2011; Van den Driessche and Watmough 2002; Diekmann and Heesterbeek 2000). There are several measures for the eradication of infectious diseases via chemical method. But, there are many problems associated with continued deployment of chemicals motivate researchers to determine a nonchemical method of disease control. Consequently, the search for non-chemical methods of disease control continues to gain importance. Some investigations (Sahoo and Poria 2013, 2014; Srinivasu et al. 2007; Srinivasu and Prasad 2010; Sahoo et al. 2016; Haque and Greenhalgh 2010; Guin et al. 2012) were done for population control in a predator-prey model providing alternative food to predator. Haque and Greenhalgh (2010) argued that alternative food source may play an important role in promoting the persistence of predator-prey systems. Guin et al. (2012) investigated the significant role of self and cross-diffusion in a prey-dependent predator-prey model in which predator has alternative source of food. Recently, Sahoo and Poria (2013) investigated the disease control aspects of alternative food to predator in predator-prey model. But, they have not consider the latent period of susceptible prey. In this paper, we formulate a diseased predator-prey model in presence of alternative food to predator considering latent period of susceptible prey.
The main aim of this paper is to analyse the role of additional food for controlling disease in a susceptibleexposed-infected prey-predator model (SEIP). The section-wise split of this paper is as follows. In "The model", we propose an epidemic model with proper assumptions in presence of additional food to predator. The local and global stability of disease free as well as predator free equilibrium states are discussed in "Dynamics of the subsystems". The local stability conditions of the steady state solution of the full system is analysed in "Dynamics of the full system". The key findings of our analytical results are verified with the help of numerical simulation in "Numerical simulation results". "Seasonally varying contact rate" devotes to study the effects of seasonally varying parameters on the system's dynamics. Finally, we draw conclusions in "Conclusions".

\section{The model}

We formulate a predator-prey model in presence of infectious disease in prey supplying additional food to predator under the following assumptions:

(a) In presence of disease, the prey population is subdivided into three classes, namely, the susceptible prey $S(t)$, exposed prey $E(t)$ (in the latent period) and infected prey $I(t)$. The density of the predator is denoted by $P(t)$ at time $t$.

(b) The susceptible prey population grows logistically with intrinsic growth rate $R$ and environmental carrying capacity $K_{0}$.

(c) We assume that a primarily infected individual becomes infectious after a latent period. The exposed species $(E)$ arise due to contacts between susceptible $(S)$ and infected prey $(I)$ at a rate of $W$, following the law of mass action.

(d) The exposed prey becomes infected at a constant rate $\mathrm{M}(>0)$ so that $1 / M$ can be regarded as the mean latent period. In the limiting case, when $M \rightarrow \infty$, the latent period is negligible and then the susceptible prey directly becomes infected.

(e) Infected prey population is not in a state of reproduction and does not compete for the resource.

(f) The interaction between predator and susceptible prey is of Holling type-II and that of between predator and exposed prey, predator and infected prey are of Holling type-I (mass action law) in absence of alternative food. This combination of functional forms are taken because the capturing rate of exposed and infected prey are easier than the susceptible prey in some ecosystem (Sahoo and Poria 2014). 
(g) Additional food of constant biomass $A$ is provided to predator. The additional food is always available in constant amounts, unaffected by consumption. This simplification is justified for many arthropod predators because they can rely on plant-provided alternative food sources such as pollen or nectar, the availability of which is unlikely to be influenced by the predator's consumption. This is in contrast to other predator-prey models which assume that the predator is a specialist that is completely dependent on the prey (Haque et al. 2009; Sahoo and Poria 2014). Almost all predators will attempt to additional when the preferred prey density becomes low.

(h) The number of encounters per predator with the additional food is proportional to the density of the additional food.

(i) The proportionality constant characterizes the ability of the predator to identify the additional food.

With the above assumptions, we formulate the following model

$\frac{d S}{d T}=S\left(1-\frac{S}{K_{0}}\right)-W S I-A_{1} \frac{S P}{B_{1}+\alpha \mu A+S}$,

$\frac{d E}{d T}=W S I-M E-A_{2} \frac{E P}{B_{1}+\alpha \mu A}-D_{1} E$,

$\frac{d I}{d T}=M E-A_{2} \frac{I P}{B_{1}+\alpha \mu A}-D_{1} I$,

$\frac{d P}{d T}=A_{1} C_{1} \frac{(S+\mu A) P}{B_{1}+\alpha \mu A+S}+C_{2} A_{2} \frac{(E+I) P}{B_{1}+\alpha \mu A}-D_{2} P$

The constants $A_{1}, A_{2}$ are maximal predation rate of predator for susceptible prey, and for both exposed and infected prey respectively. The terms $C_{1}, C_{2}$ are conversion rates of susceptible prey, both exposed and infected prey to predator respectively; $B_{1}$ is the half saturation constant for predator; $D_{1}$ and $D_{2}$ are constant death rates for $E, I$ and $P$, respectively. If $h_{1}$ represents the handling time of the predator per prey and $h_{2}$ represents the handling time of the predator per unit quantity of additional food, then $\alpha=h_{2} / h_{1}$. If the constant $e_{1}, e_{2}$ represent ability of the predator to detect the prey item and to detect additional food respectively, then $\mu=e_{2} / e_{1}$. The term $\mu A$ represents effectual additional food for the predator.

We nondimensionalize the system (1) using $s=\frac{S}{K_{0}}$, $e=\frac{E}{K_{0}}, i=\frac{I}{K_{0}}, p=\frac{P}{K_{0}}$ and $t=R T$ and the model (1) takes the following form

$$
\begin{aligned}
& \frac{d s}{d t}=s(1-s)-\gamma i s-\frac{a s p}{1+\alpha \xi+b s}, \\
& \frac{d e}{d t}=\gamma i s-m e-\frac{\beta e p}{1+\alpha \xi}-d_{1} e \\
& \frac{d i}{d t}=m e-\frac{\beta i p}{1+\alpha \xi}-d_{1} i \\
& \frac{d p}{d t}=\frac{\epsilon(s+c \xi) p}{1+\alpha \xi+b s}+\frac{\eta(e+i) p}{1+\alpha \xi}-d_{2} p,
\end{aligned}
$$

where $\quad \gamma=\frac{W K_{0}}{R}, \quad a=\frac{A_{1} K_{0}}{R B_{1}}, \quad b=\frac{K_{0}}{B_{1}}, \quad m=\frac{M}{R}, \quad \beta=\frac{A_{2} K_{0}}{R B_{1}}$, $\epsilon=\frac{C_{1} A_{1} K_{0}}{R B_{1}}, c=\frac{B_{1}}{K_{0}}, \eta=\frac{C_{2} A_{2} K_{0}}{R B_{1}}, d_{1}=\frac{D_{1}}{R}, d_{2}=\frac{D_{2}}{R}$ and $\xi=\frac{\mu A}{B_{1}}$. We analyze the system dynamics under the conditions $(\mathrm{H})$ : $0 \leq s(t) \leq 1, \quad e(t) \geq 0, \quad i(t) \geq 0, \quad p(t) \geq 0 \quad$ and $0<\epsilon<a$, $0<c<1,0 \leq \eta<\beta, d_{1}>d_{2}$. A schematic diagram of the model (2) is sketched in Fig. 1.

The constant term $\gamma$ represents the infection rate of the prey. The ratio $1 / \mathrm{m}$ represents the mean latent period and in the limiting case when $m \rightarrow \infty$, the latent period is negligible, and therefore, the susceptible prey directly becomes infected. The terms $\alpha$ and $\xi$ are the parameters which characterize the additional food. From the relation $\alpha=\frac{h_{2}}{h_{1}}$, it can be inferred that $\alpha$ is directly proportional to the handling time $h_{2}$ of the additional food. Hence the parameter $\alpha$ is proportionally related to the "quality" of the additional food. If the relation $h_{2}<h_{1}$ holds, then the predator can easily capture additional food than prey species and it implies that the additional food is of high quality. Therefore for high quality of additional food $\alpha$ is less than 1 . However, from the relation $\xi=\frac{\mu A}{B_{1}}$, it can be inferred that $\xi$ is directly proportional to the biomass of the additional food $(A)$ and thus $\xi$ is a representative of the "quantity" of the additional food that is supplied to predator (Srinivasu and Prasad 2010).

\section{Dynamics of the subsystems}

In order to understand the dynamics of full system (2), we should have a complete picture of the disease free and predator free subsystems.

1. The disease free predator-prey model [by taking $e=0$ and $i=0$ in (2)] is represented as

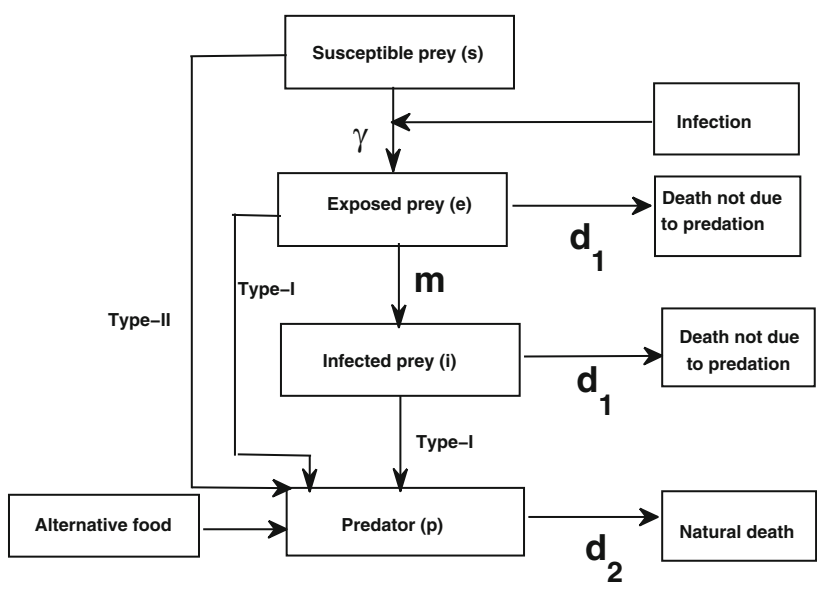

Fig. 1 Schematic diagram of the model (2) 


$$
\begin{aligned}
& \frac{d s}{d t}=s(1-s)-\frac{a s p}{1+\alpha \xi+b s}, \\
& \frac{d p}{d t}=\frac{\epsilon(s+c \xi) p}{1+\alpha \xi+b s}-d_{2} p .
\end{aligned}
$$

We introduce a disease-free demographic reproduction number for predator $R_{0}^{p}=\frac{\epsilon(1+c \xi)}{d_{2}(1+\alpha \xi+b)}$, which gives the expected number of offspring $\frac{\epsilon(1+c \xi)}{1+\alpha \xi+b}$ of an average individual predator in its lifetime $\frac{1}{d_{2}}$. The reproduction number $R_{0}^{p}$ is based on the assumptions that the susceptible prey is at unit density (i.e. $s=1$ ) and the disease is absent (i.e., $e=0$ and $i=0$ ). The value of $R_{0}^{p}<1$ indicates that the predator cannot invade susceptible prey while the value of $R_{0}^{p}>1$ indicates that the predator may invade.

2. The susceptible-exposed-infected prey model in the absence of predator [by putting $p=0$ in (2)] becomes the following form

$$
\begin{aligned}
& \frac{d s}{d t}=s(1-s)-\gamma i s, \\
& \frac{d e}{d t}=\gamma i s-m e-d_{1} e, \\
& \frac{d i}{d t}=m e-d_{1} i .
\end{aligned}
$$

The basic reproduction number of infected prey is $R_{0}^{i}=\frac{m \gamma}{d_{1}\left(m+d_{1}\right)}$, whose numerator denotes the number of secondary infections $m \gamma s^{*}=m \gamma$ per unit of time (at the locally asymptotically stable equilibrium $s^{*}=1$ ) and denominator denotes the inverse of the average infectious period $d_{1}\left(m+d_{1}\right)$. The value of $R_{0}^{i}<1$ indicates that the infection cannot invade while $R_{0}^{i}>1$ indicates that the disease can invade.

\section{Positivity and boundedness of subsystems}

Proposition 3.1 Assume that both subsystems (3) and (4) are under the Condition H. Then both subsystems are positively invariant and uniformly ultimately bounded in $\mathbb{R}_{+}^{2}$. In addition, the subsystems (3) and (4) has the following properties: $\lim _{t \rightarrow \infty} \sup \{s(t)+p(t)\} \leq \frac{1}{\theta} \quad$ and $\lim _{t \rightarrow \infty} \sup \{s(t)+e(t)+i(t)\} \leq \frac{1}{\phi}, \quad$ where $\quad \theta=$ $\min \left\{1, d_{2}-\epsilon c \xi\right\}>0, \phi=\min \left\{1, d_{1}\right\}$.

Proof For the system (3) with positive initial conditions we consider that

$$
\begin{aligned}
& w=s+p \\
& \text { i.e., } \frac{d w}{d t}=\frac{d s}{d t}+\frac{d p}{d t} .
\end{aligned}
$$

Using equations of (3), we have

$\frac{d w}{d t} \leq-\theta(s+p)-(s-1)^{2}+1$,

where $\theta=\min \left(1, d_{2}-\epsilon c \xi\right)>0$, assuming $d_{2}>\epsilon c \xi$.

Therefore, $\frac{d w}{d t}+\theta w \leq 1$.

Applying the theory of differential inequality we obtain

$0<w \leq \frac{1-e^{-\theta t}}{\theta}+w(s(0), p(0)) e^{-\theta t}$.

Thus, $\lim _{t \rightarrow \infty} \sup \{s(t)+p(t)\} \leq \frac{1}{\theta}$.

Again, for the system (4) with positive initial conditions, let us consider that

$$
\begin{aligned}
& v=s+e+i \\
& \text { i.e., } \frac{d v}{d t}=\frac{d s}{d t}+\frac{d e}{d t}+\frac{d i}{d t} .
\end{aligned}
$$

Using equations of (4), we have

$\frac{d v}{d t} \leq-\phi(s+e+i)-(s-1)^{2}+1$

where $\phi=\min \left(1, d_{1}\right)$

Therefore, $\frac{d v}{d t}+\phi v \leq 1$.

Applying the theory of differential inequality we obtain

$0<v \leq \frac{1-e^{-\phi t}}{\phi}+v(s(0), e(0), i(0)) e^{-\phi t}$.

Therefore, $\lim _{t \rightarrow \infty} \sup \{s(t)+e(t)+i(t)\} \leq \frac{1}{\phi}$.

\section{Equilibria and local stability}

The subsystem (3) has following equilibrium points: $E_{0}^{p}=(0,0), \quad E_{1}^{p}=(1,0) \quad$ and $\quad E_{2}^{p}=(\bar{s}, \bar{p}), \quad$ where $\bar{s}=\frac{\epsilon(1+c \bar{\xi})-\left(b d_{2}+\epsilon c \bar{\xi}\right) R_{0}^{p}}{R_{0}^{p}\left(\epsilon-b d_{2}\right)}, \bar{p}=\frac{1-\bar{s}}{a}(1+\alpha \xi+b \bar{s})$.

The equilibrium point $E_{2}^{p}=(\bar{s}, \bar{p})$ exists if either $\bar{s}<1$, $\epsilon<b d_{2}, R_{0}^{p}>\frac{\epsilon(1+c \xi)}{b d_{2}+\epsilon c \xi}$ or $\bar{s}<1, \epsilon>b d_{2}, R_{0}^{p}<\frac{\epsilon(1+c \xi)}{b d_{2}+\epsilon c \xi}$. We assume that $E_{2}^{p}$ exists only when $\bar{s}<1, \quad \epsilon>b d_{2}$, $R_{0}^{p}<\frac{\epsilon(1+c \xi)}{b d_{2}+\epsilon c \xi}$.

The subsystem (4) has following equilibrium points: $E_{0}^{i}=(0,0,0), \quad E_{1}^{i}=(1,0,0), \quad$ and $E_{2}^{i}=(\hat{s}, \hat{e}, \hat{i})$, where $\hat{s}=\frac{1}{R_{0}^{i}}, \hat{e}=\frac{d_{1}}{m \gamma}\left(1-\frac{1}{R_{0}^{i}}\right), \hat{i}=\frac{1}{\gamma}\left(1-\frac{1}{R_{0}^{i}}\right)$.

The equilibrium point $E_{2}^{i}$ exists if $R_{0}^{i}>1$.

Proposition 3.2 The local stability of boundary equilibrium points of both subsystems (3) and (4) are summarized in Table 1.

Proof The Jacobian matrix of the subsystem (3) at any point $(s, p)$ is given by 
$J(s, p)=\left(\begin{array}{cc}1-2 s-\frac{a p(1+\alpha \xi)}{(1+\alpha \xi+b s)^{2}} & -\frac{a s}{1+\alpha \xi+b s} \\ \frac{\epsilon p(1+\alpha \xi-b c \xi)}{(1+\alpha \xi+b s)^{2}} & \frac{\epsilon(s+c \xi)}{1+\alpha \xi+b s}-d_{2}\end{array}\right)$.

1. $E_{0}^{p}=(0,0)$ is always unstable, since one eigenvalue associated with (5) at $E_{0}^{p}$ is 1 .

2. $E_{1}^{p}=(1,0)$ is locally asymptotically stable if $R_{0}^{p}<1$ and is a saddle if $R_{0}^{p}>1$, since both the eigenvalues associated with (5) at $E_{1}^{p}$ can be represented as follows:

$\lambda_{1}=-1(<0)$,

$\lambda_{2}=d_{2}\left(R_{0}^{p}-1\right)\left\{\begin{array}{cc}<0, & \text { if } R_{0}^{p}<1, \\ >0, & \text { if } R_{0}^{p}>1 .\end{array}\right.$

3. The Jacobian matrix evaluated at $E_{2}^{p}=(\bar{s}, \bar{p})$ is

$J(\bar{s}, \bar{p})=\left(\begin{array}{cc}a_{11} & a_{12} \\ a_{21} & 0\end{array}\right)$,

where $\quad a_{11}=\frac{a b \bar{s} \bar{p}}{(1+\alpha \bar{\xi}+b \bar{s})^{2}}-\bar{s}, \quad a_{12}=-\frac{a \bar{s}}{1+\alpha \bar{\xi}+b \bar{s}}$, $a_{21}=\frac{\epsilon \bar{p}(1+\alpha \bar{\xi}-b c \xi)}{(1+\alpha \bar{\xi}+b \bar{s})^{2}}$. The characteristic equation of (6) is $\lambda^{2}-A \lambda+B=0, \quad$ where $A=a_{11}$ and $B=$ $-a_{12} a_{21}>0$. The term $A>0$ if $R_{0}^{p}>\frac{2 b \epsilon(1+\alpha \xi)}{2 b\left(b d_{2}+c \epsilon \xi\right)+\left(\epsilon-b d_{2}\right)(b-1-\alpha \xi)} \quad$ and $\quad A<0 \quad$ if $R_{0}^{p}<\frac{2 b \epsilon(1+\alpha \xi)}{2 b\left(b d_{2}+c \epsilon \xi\right)+\left(\epsilon-b d_{2}\right)(b-1-\alpha \xi)}$. The eigenvalues of (6) at $E_{2}^{p}$ are $\lambda_{1,2}=\frac{A \pm \sqrt{A^{2}-4 B}}{2}$, when $A^{2}>4 B$ or $\lambda_{1,2}=\frac{A \pm i \sqrt{4 B-A^{2}}}{2}$, when $A^{2}<4 B$. Therefore, $E_{2}^{p}=(\bar{s}, \bar{p})$ exists and is locally asymptotically stable if $1<R_{0}^{p}<$ $\min \left\{\frac{2 b \epsilon(1+\alpha \xi)}{2 b\left(b d_{2}+c \epsilon \xi\right)+\left(\epsilon-b d_{2}\right)\left(b-1-\alpha \xi^{\xi}\right)}, \frac{\epsilon(1+c \xi)}{b d_{2}+\epsilon \epsilon \xi}\right\}=\frac{2 b \epsilon}{(1+\alpha \xi)}$ $2 b\left(b d_{2}+c \epsilon \xi\right)+\left(\epsilon-b d_{2}\right)(b-1-\alpha \xi)$. The Jacobian matrix of the subsystem (4) at any point $(s, e, i)$ is given by

$$
J(s, e, i)=\left(\begin{array}{ccc}
1-2 s-\gamma i & 0 & -\gamma s \\
\gamma i & -\left(m+d_{1}\right) & \gamma s \\
0 & m & -d_{1}
\end{array}\right) .
$$

4. $E_{0}^{i}=(0,0,0)$ is always unstable, since one eigenvalue associated with (7) at $E_{0}^{i}$ is 1 .

5. $E_{1}^{i}=(1,0,0)$ is locally asymptotically stable if $R_{0}^{i}<1$ and is unstable if $R_{0}^{i}>1$, since the Jacobian matrix of the subsystem (4) at the point $E_{1}^{i}=(1,0,0)$ is given by

$J\left(E_{1}^{i}\right)=\left(\begin{array}{ccc}-1 & 0 & -\gamma \\ 0 & -\left(m+d_{1}\right) & \gamma \\ 0 & m & -d_{1}\end{array}\right)$.

The eigenvalues at $E_{1}^{i}$ are

$\lambda_{1}=-1(<0)$,

$\lambda_{2,3}=\frac{-\left(m+2 d_{1}\right) \pm \sqrt{m^{2}+4 d_{1}\left(m+d_{1}\right) R_{0}^{i}}}{2}$.

Therefore, the equilibrium point $E_{1}^{i}=(1,0,0)$ is locally asymptotically stable if $-\left(m+2 d_{1}\right)+$ $\sqrt{m^{2}+4 d_{1}\left(m+d_{1}\right) R_{0}^{i}}<0$, i.e., $\frac{m \gamma}{d_{1}\left(m+d_{1}\right)}<1$ and thus $E_{1}^{i}$ is locally asymptotically stable if $R_{0}^{i}<1$, otherwise $E_{1}^{i}$ is unstable if $R_{0}^{i}>1$.

6. The Jacobian matrix evaluated at $E_{2}^{i}=(\hat{s}, \hat{e}, \hat{p})$ is

$J\left(E_{2}^{i}\right)=\left(\begin{array}{ccc}1-2 \hat{s}-\gamma \hat{i} & 0 & -\gamma \hat{s} \\ \gamma \hat{i} & -\left(m+d_{1}\right) & \gamma \hat{s} \\ 0 & m & -d_{1}\end{array}\right)$.

The characteristic equation of (8) is $\lambda^{3}+T_{1} \lambda^{2}+T_{2} \lambda+T_{3}=0, \quad$ where $T_{1}=m+2 d_{1}+$ $2 \hat{s}+\gamma \hat{i}-1=m+2 d_{1}+\frac{1}{R_{0}^{i}}$,

$T_{2}=-\left[d_{1}\left(m+d_{1}\right)-m \hat{s} \gamma+\left(m+2 d_{1}\right)(1-2 \hat{s}-\gamma \hat{i})\right]=\frac{m+2 d_{1}}{R_{0}^{i}}$, $T_{3}=\left[\gamma^{2} m \hat{i} \hat{s}-(1-2 \hat{s}-\gamma \hat{i})\left(d_{1}\left(m+d_{1}\right)-m \gamma \hat{s}\right)\right]=\frac{\gamma m}{R_{0}^{i}}\left(1-\frac{1}{R_{0}^{i}}\right)$. if $R_{0}^{i}<\frac{m+2 d_{1}+\gamma m}{\gamma m-\left(m+2 d_{1}\right)^{2}}$. Hence, the sufficient conditions for the positive equilibrium point $E_{2}^{i}$ of the subsystem (4) is to be locally asymptotically stable if $1<R_{0}^{i}<\frac{m+2 d_{1}+\gamma m}{\gamma m-\left(m+2 d_{1}\right)^{2}}$ holds.
Table 1 The local stability conditions of boundary and interior equilibrium points for both subsystems (3) and (4)

\begin{tabular}{ll}
\hline Equilibrium points & Stability conditions \\
\hline$E_{0}^{p}$ & Always unstable \\
$E_{1}^{p}$ & Locally asymptotically stable if $R_{0}^{p}<1$; saddle if $R_{0}^{p}>1$ \\
$E_{2}^{p}$ & Locally asymptotically stable if $1<R_{0}^{p}<\frac{2 b \epsilon(1+\alpha \xi)}{2 b\left(b d_{2}+c \epsilon \xi\right)+\left(\epsilon-b d_{2}\right)(b-1-\alpha \xi)}$ \\
$E_{0}^{i}$ & Always unstable \\
$E_{1}^{i}$ & Locally asymptotically stable if $R_{0}^{i}<1 ;$ unstable if $R_{0}^{i}>1$ \\
$E_{2}^{i}$ & Locally asymptotically stable if $1<R_{0}^{i}<\frac{m+2 d_{1}+\gamma m}{\gamma m-\left(m+2 d_{1}\right)^{2}}$ \\
\hline
\end{tabular}


Note Since we have seen that for $R_{0}^{i}>1$, the endemic equilibrium $E_{2}^{i}$ is locally asymptotically stable but the disease free equilibrium is unstable. Therefore, the subsystem (4) will be never disease free if $R_{0}^{i}>1$.

\section{Global stability}

Theorem 3.1 The subsystem (3) is globally asymptotically stable at the equilibrium point $E_{2}^{p}=(\bar{s}, \bar{p})$ in the region $\Upsilon_{1}=\left\{(s, p): 1<\frac{s}{\bar{s}}<\frac{p}{\bar{p}}\right\}$.

Proof We choose a Lyapunov function $W_{1}(s, p)$ as the following:

$$
W_{1}(s, p)=\int_{\bar{s}}^{s} \frac{s-\bar{s}}{s} d s+\frac{a}{\epsilon} \int_{\bar{p}}^{p} \frac{p-\bar{p}}{p} d p .
$$

Calculating time derivative of the equation (9) along the solutions of the system (3) gives

$$
\begin{aligned}
\frac{d W_{1}}{d t}= & \frac{(s-\bar{s})}{s} \frac{d s}{d t}+\frac{a(p-\bar{p})}{\epsilon} \frac{d p}{d t}, \\
= & (s-\bar{s})\left\{-(s-\bar{s})-a\left(\frac{p}{1+\alpha \xi+b s}-\frac{\bar{p}}{1+\alpha \xi+b \bar{s}}\right)\right\} \\
& +\frac{a}{\epsilon}(p-\bar{p})\left\{\epsilon\left(\frac{s}{1+\alpha \xi+b s}-\frac{\bar{s}}{1+\alpha \xi+b \bar{s}}\right)\right. \\
& \left.+\epsilon c \xi\left(\frac{1}{1+\alpha \xi+b s}-\frac{1}{1+\alpha \xi+b \bar{s}}\right)\right\}, \\
= & -(s-\bar{s})^{2}-\frac{a c \xi b(s-\bar{s})(p-\bar{p})}{(1+\alpha \bar{\xi}+b s)(1+\alpha \xi+b \bar{s})} \\
& -\frac{a b(s-\bar{s})(p \bar{s}-\bar{p} s)}{(1+\alpha \bar{\xi}+b s)(1+\alpha \bar{\xi}+b \bar{s})} .
\end{aligned}
$$

It is clear that $\frac{d W_{1}}{d t}<0$ in $\Upsilon_{1}$. Hence the proof is completed.

Theorem 3.2 The subsystem (4) is globally asymptotically stable at the equilibrium point $E_{2}^{i}=\left(s^{*}, e^{*}, i^{*}\right)$ in the region $\Upsilon_{2}=\left\{(s, e, i): \gamma>\frac{m\left(i-i^{*}\right)\left(e-e^{*}\right)}{\left(s-s^{*}\right)\left(i-i^{*}\right)-\left(i s-i^{*} s^{*}\right)\left(e-e^{*}\right)}\right\}$.

Proof We choose a Lyapunov function $W_{2}(s, e, i)$ defined as follows:

$W_{2}(s, e, i)=\int_{s^{*}}^{s} \frac{s-s^{*}}{s} d s+\int_{e^{*}}^{e} \frac{e-e^{*}}{e} d e+\int_{i^{*}}^{i} \frac{i-i^{*}}{i} d i$.

Calculating time derivative of the equation (10) along the solutions of the system (4) gives

$$
\begin{aligned}
\frac{d W_{2}}{d t}= & \frac{\left(s-s^{*}\right)}{s} \frac{d s}{d t}+\frac{\left(e-e^{*}\right)}{e} \frac{d e}{d t}+\frac{\left(i-i^{*}\right)}{i} \frac{d i}{d t}, \\
= & \left(s-s^{*}\right)\left\{-\left(s-s^{*}\right)-\gamma\left(i-i^{*}\right)\right\}+\left(e-e^{*}\right) \\
& \times\left\{\frac{\gamma}{e}\left(i s-i^{*} s^{*}\right)\right\}+\left(i-i^{*}\right)\left\{\frac{m}{i}\left(e-e^{*}\right)\right\},
\end{aligned}
$$

$$
\begin{aligned}
=- & \left(s-s^{*}\right)^{2}-\gamma\left(s-s^{*}\right)\left(i-i^{*}\right)+\gamma\left(i s-i^{*} s^{*}\right) \\
& \times\left(1-\frac{e^{*}}{e}\right)+m\left(e-e^{*}\right)\left(1-\frac{i^{*}}{i}\right) .
\end{aligned}
$$

It is clear that $\frac{d W_{2}}{d t}<0$ in $\Upsilon_{2}$. Hence the theorem is proved.

\section{Dynamics of the full system}

\section{Equilibria}

After obtaining the dynamics of the subsystems (3) and (4) in the previous section, we now study the dynamics of the full system (2). We start with determining the boundary and endemic equilibrium points and their stability of the full system (2). It is easy to check that the system (2) possesses the following equilibrium points:

1. The trivial equilibrium point is $E_{T}=(0,0,0,0)$.

2. The disease free equilibrium point is $E_{D F}=(1,0,0,0)$.

3. The disease free boundary equilibrium point is $E_{D F B}=(\bar{s}, 0,0, \bar{p}), \quad$ where $\quad \bar{s}=\frac{d_{2}+\xi\left(d_{2} \alpha-\epsilon c\right)}{\epsilon-b d_{2}}$, $\bar{p}=\frac{\epsilon}{a}\left[\frac{\epsilon-d_{2}-b d_{2}-\xi \xi\left(\epsilon c+d_{2} \alpha\right)}{\left(\epsilon-b d_{2}\right)^{2}}\right](1+\alpha \xi-b c \xi) . E_{D F B}$ exists if $d_{2}+\xi\left(d_{2} \alpha-\epsilon c\right)>0 \quad$ and $\epsilon-d_{2}-b d_{2}>\xi\left(\epsilon c+d_{2} \alpha\right)$. Therefore, the existence criteria of disease free boundary equilibrium point is $\frac{d_{2}}{\epsilon c-d_{2} \alpha}<\xi<\frac{\epsilon-d_{2}-b d_{2}}{\epsilon c+d_{2} \alpha}$, which depends on quality of additional food.

4. The endemic equilibrium point is $E^{*}=\left(s^{*}, e^{*}, i^{*}, p^{*}\right)$. Let us consider the quantities $k_{1}=\frac{p^{*}}{1+\alpha \xi^{*}+b s^{*}}$, and $k_{2}=\frac{p^{*}}{1+\alpha \xi^{*}}$. Using these quantities, we obtain $s^{*}=\frac{m+d_{1}+\beta k_{2}}{\left(\beta k_{2}+d_{1}\right) \gamma}, \quad e^{*}=\frac{1}{\eta k_{2}}\left[d_{2} p^{*}-\epsilon\left(s^{*}+c \xi\right) k_{1}-\eta i^{*} k_{2}\right]$, $i^{*}=\frac{m e^{*}}{\beta k_{2}+d_{1}}$ and $p^{*}=\frac{1+\alpha \xi+b s^{*}}{a}\left(1-s^{*}-\gamma i^{*}\right)$. The endemic equilibrium point $E^{*}$ exists if $d_{2} p^{*}>\epsilon\left(s^{*}+\right.$ $c \xi) k_{1}+\eta i^{*} k_{2}$ and $1>s^{*}+\gamma i^{*}$.

It is clear that existence of equilibrium points $E_{D F}, E_{D F B}$ and $E^{*}$ depend on infection rate $(\gamma)$, the quality $(\alpha)$ and quantity $(\xi)$ of additional food.

\section{Local stability of equilibria}

Theorem 4.1 The trivial equilibrium point $E_{T}$ is always unstable. The disease free equilibrium point $E_{D F}$ is stable if $R_{0}^{i}<1$ and $R_{0}^{p}<1$, otherwise unstable.

Proof The Jacobian matrix $J\left(E_{T}\right)$ at $E_{T}$ is given by 


$$
J\left(E_{T}\right)=\left(\begin{array}{cccc}
1 & 0 & 0 & 0 \\
0 & -\left(m+d_{1}\right) & 0 & 0 \\
0 & m & -d_{1} & 0 \\
0 & 0 & 0 & \frac{\epsilon c \xi}{1+\alpha \xi}-d_{2}
\end{array}\right) .
$$

$E_{T}$ is always unstable, since $J\left(E_{T}\right)$ has one eigenvalue 1 .

The Jacobian matrix $J\left(E_{D F}\right)$ at $E_{D F}$ is given by

$$
J\left(E_{D F}\right)=\left(\begin{array}{cccc}
-1 & 0 & -\gamma & -\frac{a}{1+\alpha \xi+b} \\
0 & -\left(m+d_{1}\right) & \gamma & 0 \\
0 & m & -d_{1} & 0 \\
0 & 0 & 0 & \frac{\epsilon(1+c \xi)}{1+\alpha \xi+b}-d_{2}
\end{array}\right) .
$$

The eigenvalues of the Jacobian matrix $J\left(E_{D F}\right)$ are -1 , $\frac{\epsilon(1+c \xi)}{1+\alpha \xi+b}-d_{2}$ and the roots of the equation $\lambda^{2}+\left(m+2 d_{1}\right) \lambda+d_{1}\left(m+d_{1}\right)\left(1-R_{0}^{i}\right)=0$. The equilibrium point $E_{D F}$ is stable if $R_{0}^{i}<1$ and $\frac{\epsilon(1+c \xi)}{1+\alpha \xi+b}<d_{2}$ i.e., $R_{0}^{p}<1$, otherwise $E_{D F}$ is unstable.

Theorem 4.2 The disease free boundary equilibrium point $E_{D F B}$ of the system (2) is locally stable if the conditions $\quad \Omega_{1}>0, \Omega_{1} \Omega_{2}-\Omega_{3}>0, \quad \Omega_{3}\left(\Omega_{1} \Omega_{2}-\Omega_{3}\right)-$ $\Omega_{4} \Omega_{1}^{2}>0$ hold, where $\Omega$ 's are given in the proof of this theorem.

Proof The Jacobian matrix $J\left(E_{D F B}\right)$ at disease free equilibrium point $E_{D F B}$ is given by

$J\left(E_{D F B}\right)=\left(\begin{array}{llll}a_{11} & a_{12} & a_{13} & a_{14} \\ a_{21} & a_{22} & a_{23} & a_{24} \\ a_{31} & a_{32} & a_{33} & a_{34} \\ a_{41} & a_{42} & a_{43} & a_{44}\end{array}\right)$,

where, $\quad a_{11}=\frac{a b \bar{s} \bar{p}}{(1+\alpha \bar{\xi}+b \bar{s})^{2}}-\bar{s}, \quad a_{12}=0, \quad a_{13}=-\gamma \bar{s}$, $a_{14}=-\frac{a \bar{s}}{1+\alpha \cdot \bar{\xi}+b \bar{s}}, \quad a_{21}=0, \quad a_{22}=-m-\frac{\beta \bar{p}}{1+\alpha \bar{\xi}+b \bar{s}}-d_{1}$, $a_{23}=\gamma \bar{s}, \quad a_{24}=0, a_{31}=0, a_{32}=m, a_{33}=-\frac{\beta \bar{p}}{1+\alpha \bar{\xi}}-d_{1}$, $a_{34}=0, a_{41}=\frac{\epsilon \bar{p}(1+\alpha \bar{\xi}-b c \xi)}{(1+\alpha \bar{\xi}+b \bar{s})^{2}}, a_{42}=\frac{\eta \bar{p}}{1+\alpha \bar{\xi}}, a_{43}=\frac{\eta \bar{p}}{1+\alpha \bar{\xi}}, a_{44}=0$.

The characteristic equation of Jacobian matrix $J\left(E_{D F B}\right)$ is given by $\lambda^{4}+\Omega_{1} \lambda^{3}+\Omega_{2} \lambda^{2}+\Omega_{3} \lambda+\Omega_{4}=0$,

where,

$$
\begin{aligned}
\Omega_{1}= & -\left[a_{11}+a_{22}+a_{33}\right], \\
\Omega_{2}= & {\left[a_{11} a_{33}+a_{22} a_{33}+a_{11} a_{22}-a_{23} a_{32}-a_{14} a_{41}\right], } \\
\Omega_{3}= & -\left[a_{33} a_{11} a_{22}-a_{34} a_{43}\left(a_{11}+a_{22}\right)-a_{32} a_{11} a_{23}\right. \\
& \left.+a_{31}\left(a_{12} a_{23}-a_{13} a_{22}\right)-a_{41} a_{14} a_{23}-a_{14} a_{33} a_{41}\right], \\
\Omega_{4}= & -a_{14} a_{23}\left(a_{31} a_{42}-a_{32} a_{41}\right) .
\end{aligned}
$$

The disease free boundary equilibrium point $E_{D F B}$ for the system (2) is locally asymptotically stable if the conditions
$\Omega_{1}>0, \Omega_{1} \Omega_{2}-\Omega_{3}>0, \quad \Omega_{3}\left(\Omega_{1} \Omega_{2}-\Omega_{3}\right)-\Omega_{4} \Omega_{1}^{2}>0$ hold.

Theorem 4.3 The endemic equilibrium point $E^{*}$ of the system (2) is locally stable if the conditions $\sigma_{1}>0, \sigma_{1} \sigma_{2}-\sigma_{3}>0, \sigma_{3}\left(\sigma_{1} \sigma_{2}-\sigma_{3}\right)-\sigma_{4} \sigma_{1}^{2}>0$ hold, where $\sigma$ 's are given in the proof of this theorem.

Proof The Jacobian matrix $J\left(E^{*}\right)$ at the endemic equilibrium point $E^{*}\left(s^{*}, e^{*}, i^{*}, p^{*}\right)$ is

$J\left(E^{*}\right)=\left(\begin{array}{llll}A_{11} & A_{12} & A_{13} & A_{14} \\ A_{21} & A_{22} & A_{23} & A_{24} \\ A_{31} & A_{32} & A_{33} & A_{34} \\ A_{41} & A_{42} & A_{43} & A_{44}\end{array}\right)$,

where, $\quad A_{11}=\frac{a b s^{*} p^{*}}{\left(1+\alpha \alpha^{\xi}+b s^{*}\right)^{2}}-s^{*}, \quad A_{12}=0, \quad A_{13}=-\gamma s^{*}$, $A_{14}=-\frac{a s^{*}}{1+\alpha \xi^{*}+b s^{*}}, \quad A_{21}=\gamma i^{*}, \quad A_{22}=-m-\frac{\beta p^{*}}{1+\alpha \xi}-d_{1}$, $A_{23}=\gamma s^{*}, \quad A_{24}=-\frac{\beta e^{*}}{1+\alpha \xi}, \quad A_{31}=0, \quad A_{32}=m$, $A_{33}=-\frac{\beta p^{*}}{1+\alpha \xi}-d_{1}, \quad A_{34}=-\frac{\beta i^{*}}{1+\alpha \xi^{*}}, \quad A_{41}=\frac{\epsilon p^{*}(1+\alpha \xi-b c \xi)}{\left(1+\alpha \xi+b s^{*}\right)^{2}}$, $A_{42}=\frac{\eta p^{*}}{1+\alpha \xi^{\prime}}, A_{43}=\frac{\eta p^{*}}{1+\alpha \xi^{\xi}}, A_{44}=0$.

The characteristic equation of the Jacobian matrix $J\left(E^{*}\right)$ is $\lambda^{4}+\sigma_{1} \lambda^{3}+\sigma_{2} \lambda^{2}+\sigma_{3} \lambda+\sigma_{4}=0$,

where,

$$
\begin{aligned}
& \sigma_{1}=- {\left[A_{11}+A_{22}+A_{33}\right], } \\
& \sigma_{2}=\left[A_{11} A_{33}+A_{22} A_{33}+A_{11} A_{22}-A_{23} A_{32}-A_{24} A_{42}\right. \\
&\left.+A_{24} A_{32}-A_{34} A_{43}-A_{14} A_{41}\right] \\
& \sigma_{3}=- {\left[-\left(A_{11}+A_{22}\right) A_{34} A_{43}+A_{11} A_{22} A_{33}\right.} \\
&-A_{32}\left(A_{11} A_{23}-A 13 A_{21}\right)+A_{23} A_{34} A_{42} \\
&-\left(A_{42}-A_{32}\right)\left(A_{11} A_{24}-A_{14} A_{21}\right)-A_{24} A_{42} A_{33} \\
&\left.+A_{13} A_{41} A_{34}+A_{41}\left(A_{12} A_{24}-A_{14} A_{23}\right)-A_{14} A_{33} A_{41}\right], \\
& \sigma_{4}=[-A_{11} A_{22} A_{34} A_{43}+\left(A_{11} A_{23}-A_{13} A_{21}\right) A_{34} A_{42} \\
&-A_{42} A_{33}\left(A_{11} A_{24}-A_{14} A_{21}\right) \\
&-A_{41} A_{34}\left(A_{12} A_{23}-A_{13} A_{22}\right) \\
&\left.-A_{32} A_{41}\left(A_{13} A_{24}-A_{14} A_{23}\right)\right] .
\end{aligned}
$$

The endemic equilibrium point $E^{*}$ of the system (2) is locally asymptotically stable if the conditions $\sigma_{1}>0, \sigma_{1} \sigma_{2}-\sigma_{3}>0, \sigma_{3}\left(\sigma_{1} \sigma_{2}-\sigma_{3}\right)-\sigma_{4} \sigma_{1}^{2}>0$ hold.

We observe that the stability conditions of all the equilibrium points depend on the parameters $\xi$ and $\alpha$.

\section{Numerical simulation results}

In this section, we perform numerical simulations with a set of parameter values taken from either field or experimental data (see, Table 2) which remains fixed for all numerical simulations. In our numerical simulations we 
Table 2 Ranges of parameters with their sources

\begin{tabular}{llll}
\hline Parameters & Descriptions & Ranges & Default value \\
\hline $\mathrm{a}$ & Attack rate of susceptible prey & $0.61-6.087$ (Taylor and Walters 2010) & 2.0 \\
$\beta$ & Attack rate of exposed and infected prey & $0-0.5$ (Steele and Henderson 1992) & 0.12 \\
$\mathrm{~b}$ & Half saturation constant & $2-6.2$ (Hastings and Powell 1991) & 3 \\
$\mathrm{~m}$ & Rate of conversion from exposed to infected prey & - & 0.95 \\
$\epsilon$ & Conversion rate of susceptible prey & $0.05-0.33$ (Butzel and Bolten 1968) & 0.25 \\
$\eta$ & Conversion rate of exposed and infected prey & $0.05-0.33$ (Butzel and Bolten 1968) & 0.05 \\
$\mathrm{c}$ & Conversion rate of additional food & $0<\mathrm{c}<1$ & 0.3 \\
$d_{1}$ & Exposed and infected prey mortality rate & $0.04-0.16$ (Cheney 2004; Evans et al. 2006) & 0.08 \\
$d_{2}$ & Predator mortality rate & $0.04-0.16$ (Cheney 2004; Evans et al. 2006) & 0.04 \\
\hline
\end{tabular}

(a)

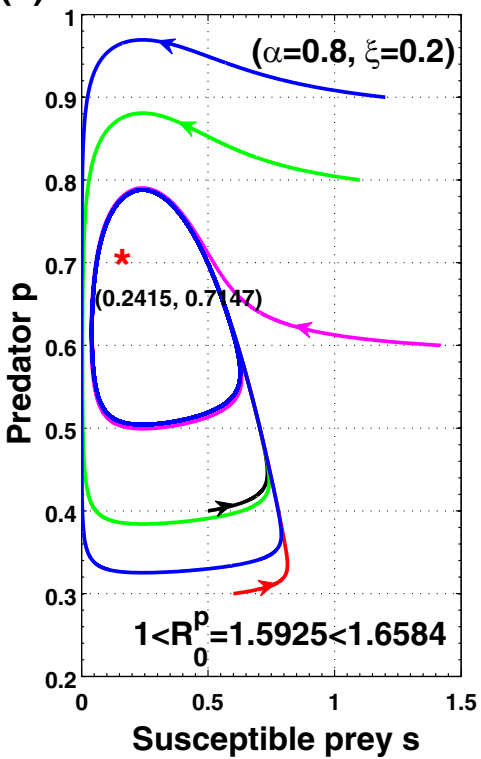

(b)

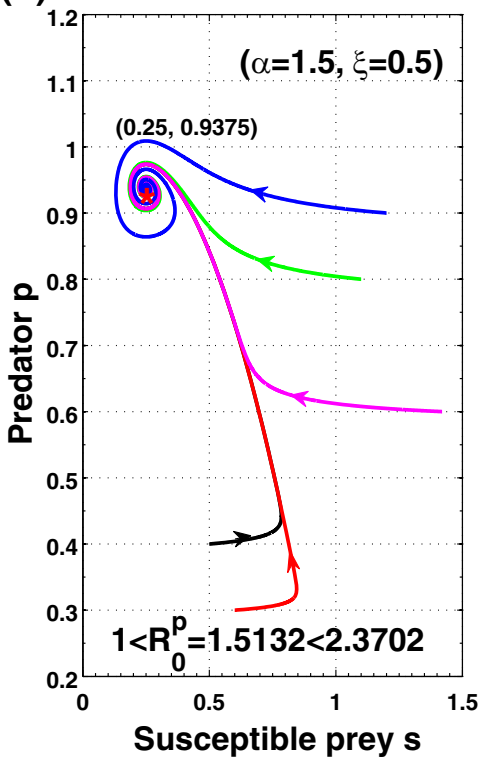

(c)

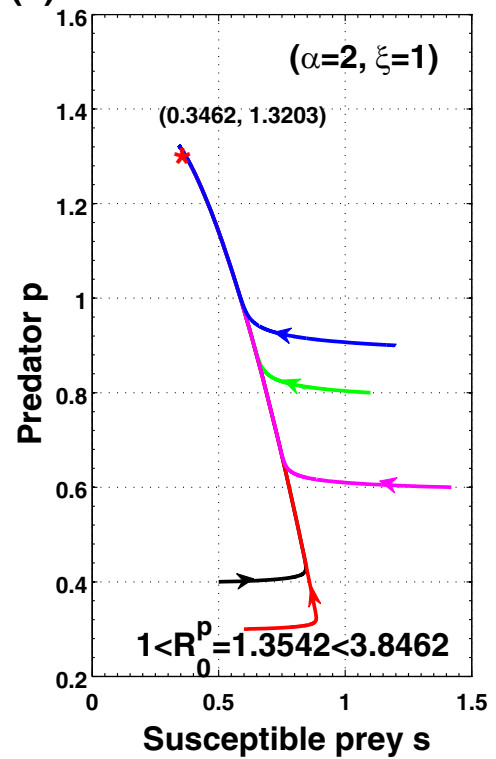

Fig. 2 Phase plane trajectories corresponding to different initial conditions $I_{1}=(0.5,0.4)$ (black line), $I_{2}=(1.1,0.8)$ (green line), $I_{3}=(0.6,0.3)$ (red line $), I_{4}=(1.42,0.6)$ (magenta line $)$ and $I_{5}=$

vary the infection rate $(\gamma)$, the quality of additional food $(\alpha)$ and the quantity of additional food $(\xi)$ within specified ranges.

The path of the disease free subsystem (3) is presented in Fig. 2 starting from different initial conditions $I_{1}=$ $(0.5,0.4)$ (black line), $I_{2}=(1.1,0.8)$ (green line), $I_{3}=$ $(0.6,0.3)$ (red line), $I_{4}=(1.42,0.6)$ (magenta line) and $I_{5}=(1.2,0.9)$ (blue line). From Fig. $2 \mathrm{a}$, it is evident that the state of the system (3) cannot reach the equilibrium point $(0.2415,0.7147)$ but tends to a limit cycle around the target. Therefore, the system (3) has a asymptotically stable limit cycle around $(0.2415,0.7147)$. Notice that if we choose initial condition very close to $(0.2415,0.7147)$, then it reaches the target state, since $1<R_{0}^{p}=$ $1.5925<1.6584$, satisfies the stability condition of
$(1.2,0.9)$ (blue line) of the subsystem (3) for $\mathbf{a} \alpha=0.8, \xi=0.2$, b $\alpha=1.5, \xi=0.5$ and $\mathbf{c} \alpha=2, \xi=1$. It depicts the global stability of the subsystem (3) about interior equilibrium points

Proposition 3.2. If we supply additional food $\alpha=1.5$ and $\xi=0.5$ to predator, the path of the system (3) reaches to the target $(0.25,0.9375)$ satisfying the stability condition $1<R_{0}^{p}=1.5132<2.3702$ and it is shown in Fig. 2b. Figure $2 \mathrm{c}$ presents that the system (3) reaches to $(0.3462,1.3202)$ satisfying the condition $1<R_{0}^{p}=$ $1.3542<3.8462$ for $\alpha=2, \xi=1$. From, Fig. $2 \mathrm{~b}$ and $\mathrm{c}$, the global stability nature of the system (3) at the fixed point is clear. Therefore, basin of attraction of the equilibrium point increases in presence of additional food.

The path of the predator free subsystem (4) is displayed in Fig. 3 with initial conditions $I_{1}=(1.8,0.8,0.3)$ (black line $), I_{2}=(2.8,1.2,0.8)($ green line $), I_{3}=(3.6,1.5,1)(\mathrm{red}$ line $), \quad I_{4}=(1.6,0.8,0.5) \quad($ magenta line $)$ and $I_{5}=$ $(2.2,1.1,0.8)$ (blue line). Starting from these initial 
(a)

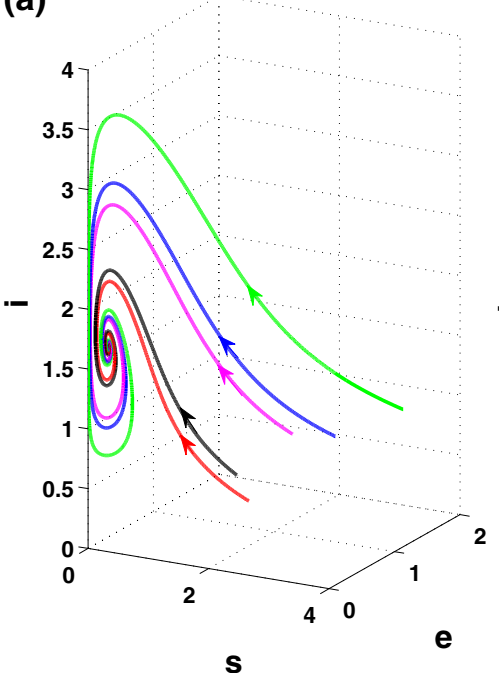

(b)

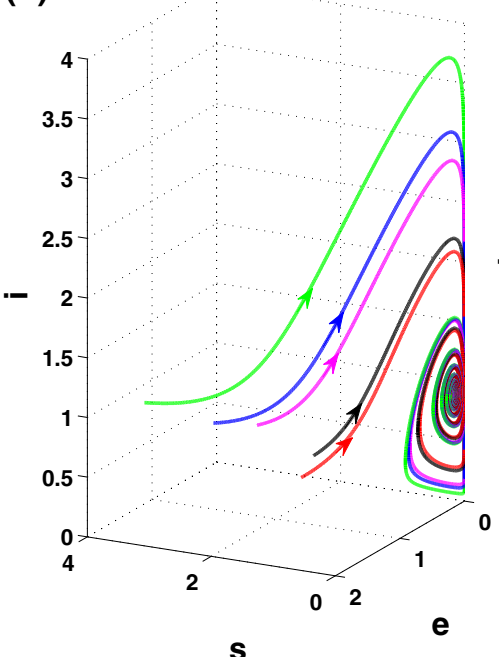

(c)

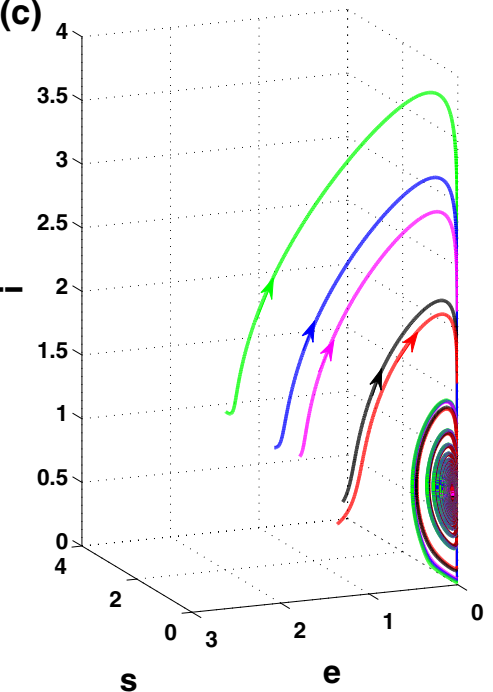

Fig. 3 Phase plane trajectories corresponding to different initial conditions $I_{1}=(1.8,0.8,0.3)$ (red line), $I_{2}=(2.8,1.2,0.8)$ (blue line $), I_{3}=(3.6,1.5,1)$ (green line $), I_{4}=(1.6,0.8,0.5)$ (black line $)$ and $I_{5}=(2.2,1.1,0.8)$ (magenta line) of the subsystem (4) for a $\gamma=0.5$, $\mathbf{b} \gamma=1$ and $\mathbf{c} \gamma=1.3$. It depicts the global stability of the subsystem (4) about interior equilibrium points (a)

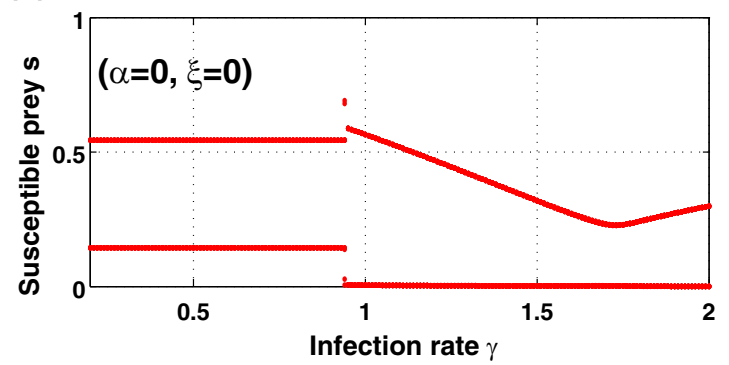

(c)

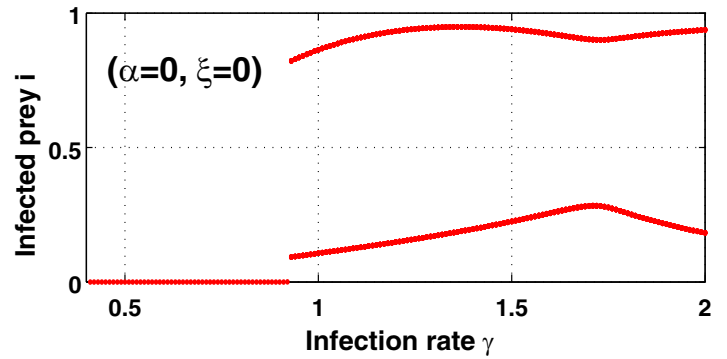

(b)

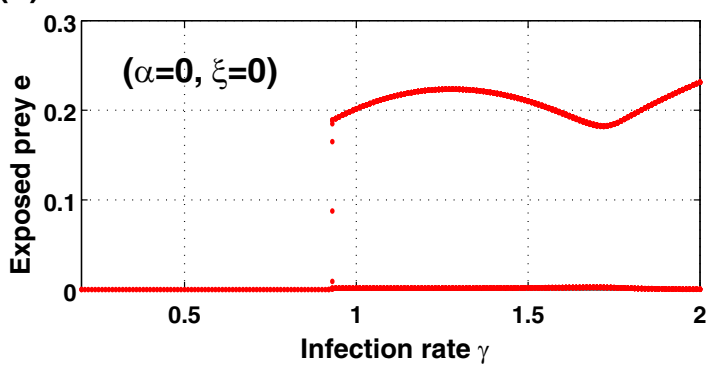

(d)

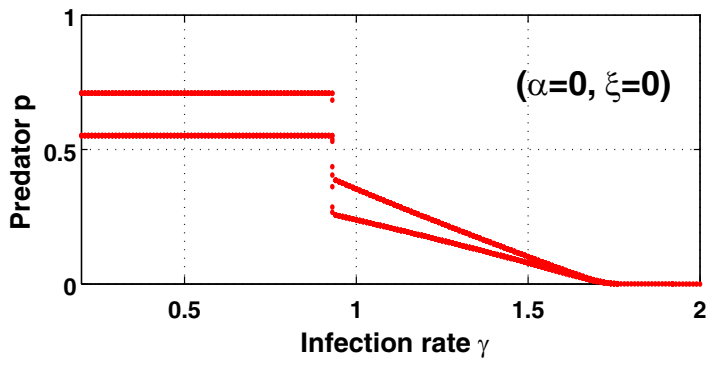

Fig. 4 Bifurcation diagram of the system (2) with respect to infection rate $\gamma$ of the prey in absence additional food i.e., for $\alpha=0$ and $\xi=0$

solutions, from Fig. 3, it is evident that the system (4) reaches to the target equilibrium point $(\hat{s}, \hat{e}, \hat{i}) \equiv$ $(0.0035,0.1678,1.9931)$ for $\gamma=0.5,(\hat{s}, \hat{e}, \hat{i}) \equiv(0.1735$, $0.0696,0.8265)$ for $\gamma=1$ and $(\hat{s}, \hat{e}, \hat{i}) \equiv(0.0578,0.0610$, $0.7248)$ for $\gamma=1.3$. From Fig. 3, we predict that the predator free equilibrium point of the system (4) has globally stable dynamics for some infection rate.

The bifurcation diagram of the system (2), in absence of additional food (i.e., $\alpha=0, \xi=0$ ), with respect to infection rate $(\gamma)$ in the range $0.2 \leq \gamma \leq 2$ is presented in Fig. 4 .
Figure 4 shows that the exposed and infected prey individuals extinct (i.e., the system becomes disease free) when $0.2 \leq \gamma \leq 0.94$ and the susceptible prey and predator individuals have limit cycle oscillations. But, for $0.94<\gamma \leq 2$, the susceptible prey, exposed prey and infected prey have limit cycle oscillations. Within $0.94<\gamma<1.75$, the predator species survives, but for $1.75 \leq \gamma \leq 2$, the predator species extinct. Therefore, susceptible prey, exposed prey and infected preys are not sufficient for persistence of predator species for high infection rate. 

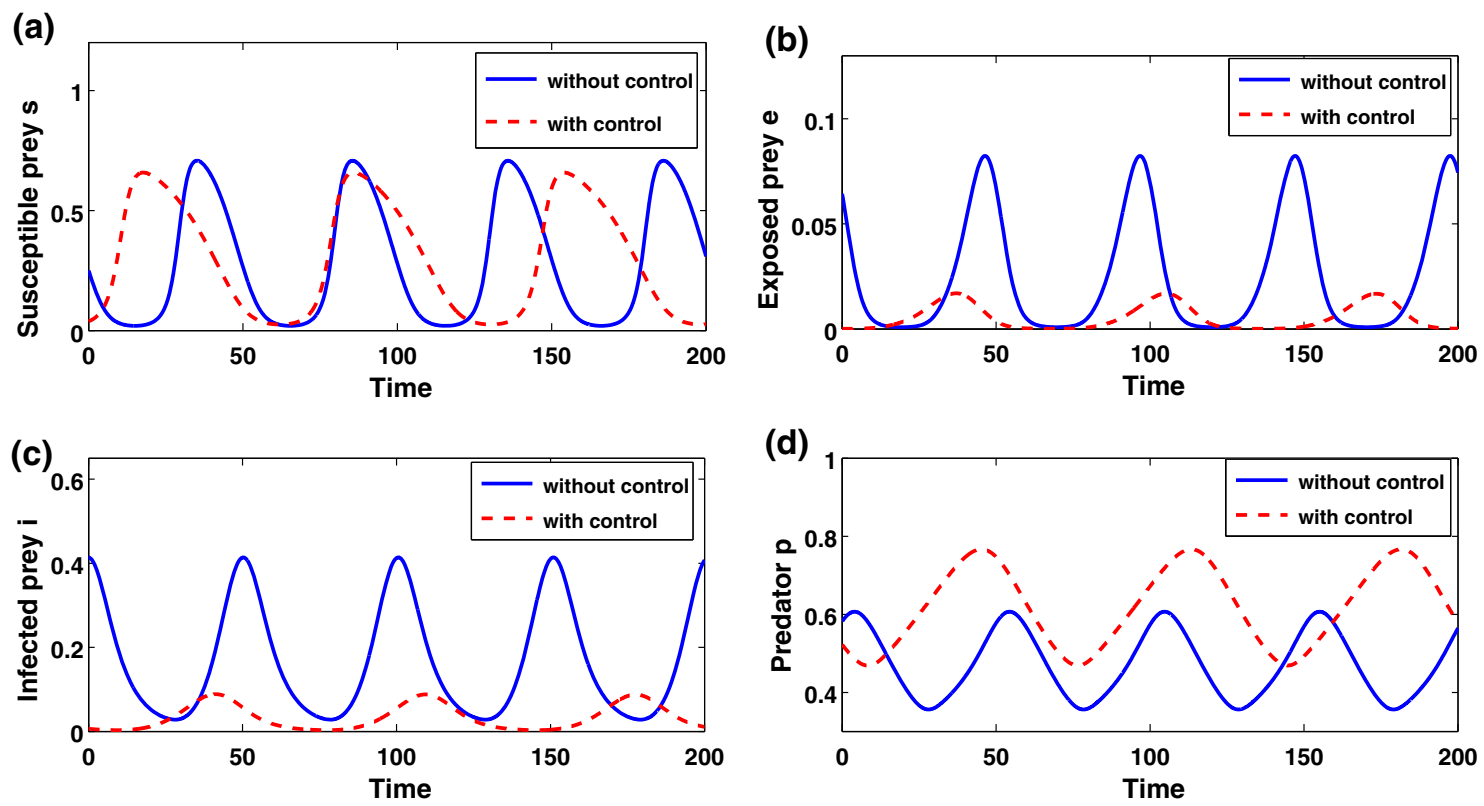

Fig. 5 Graph for the state variables of the system (2) with and without control for infection rate $\gamma=0.6$ and control parameters $\alpha=0.8, \xi=0.2$

(a)

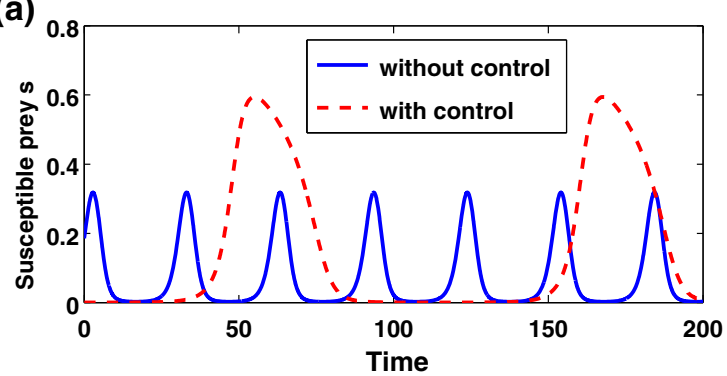

(c)

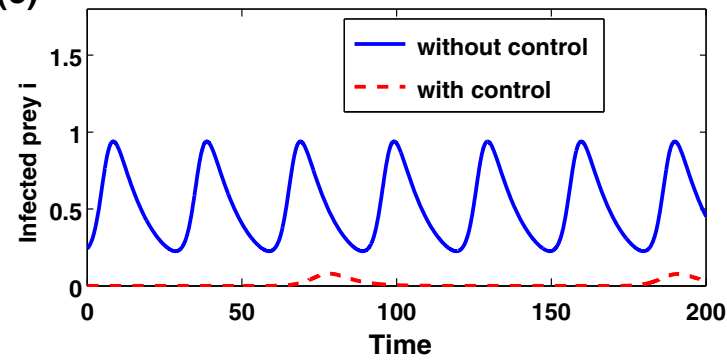

(b)

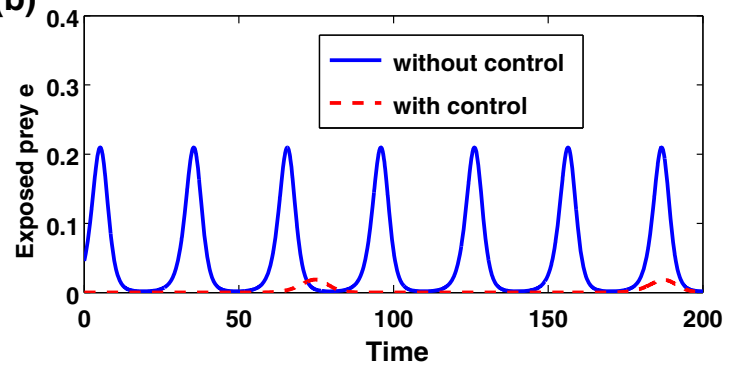

(d)

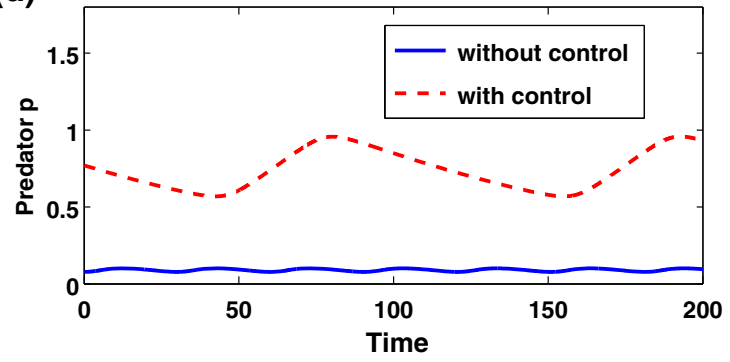

Fig. 6 Graph for the state variables of the system (2) with and without control for infection rate $\gamma=1.5$ and control parameters $\alpha=0.8, \xi=0.5$

We draw the time evolution of susceptible (s), exposed (e), infected (i) and predator (p) populations in Fig. 5 in presence as well as in absence of additional food (control variable) for fixed infection rate at $\gamma=0.6$. We observe that the application of additional food reduces the number of susceptible prey, exposed prey as well as infected prey than those populations in absence of additional food. Again from Fig. 5, it is easy to see that the predator population also affected very much due to the presence of the additional food. This happens because in presence of additional food the infected and exposed prey population reduces significantly. For higher infection rate $\gamma=1.5>0.6$, the solution curves of state variables are presented in Fig. 6 . From Figs. 5, 6, we may point out that application of additional food reduces the number of exposed as well as infected populations. Therefore, we observe that additional food to predator plays a vital role to control infection in a food chain model with infected prey.

Now, we investigate the effects of variation of either quality or quantity of additional food in the system (2). 

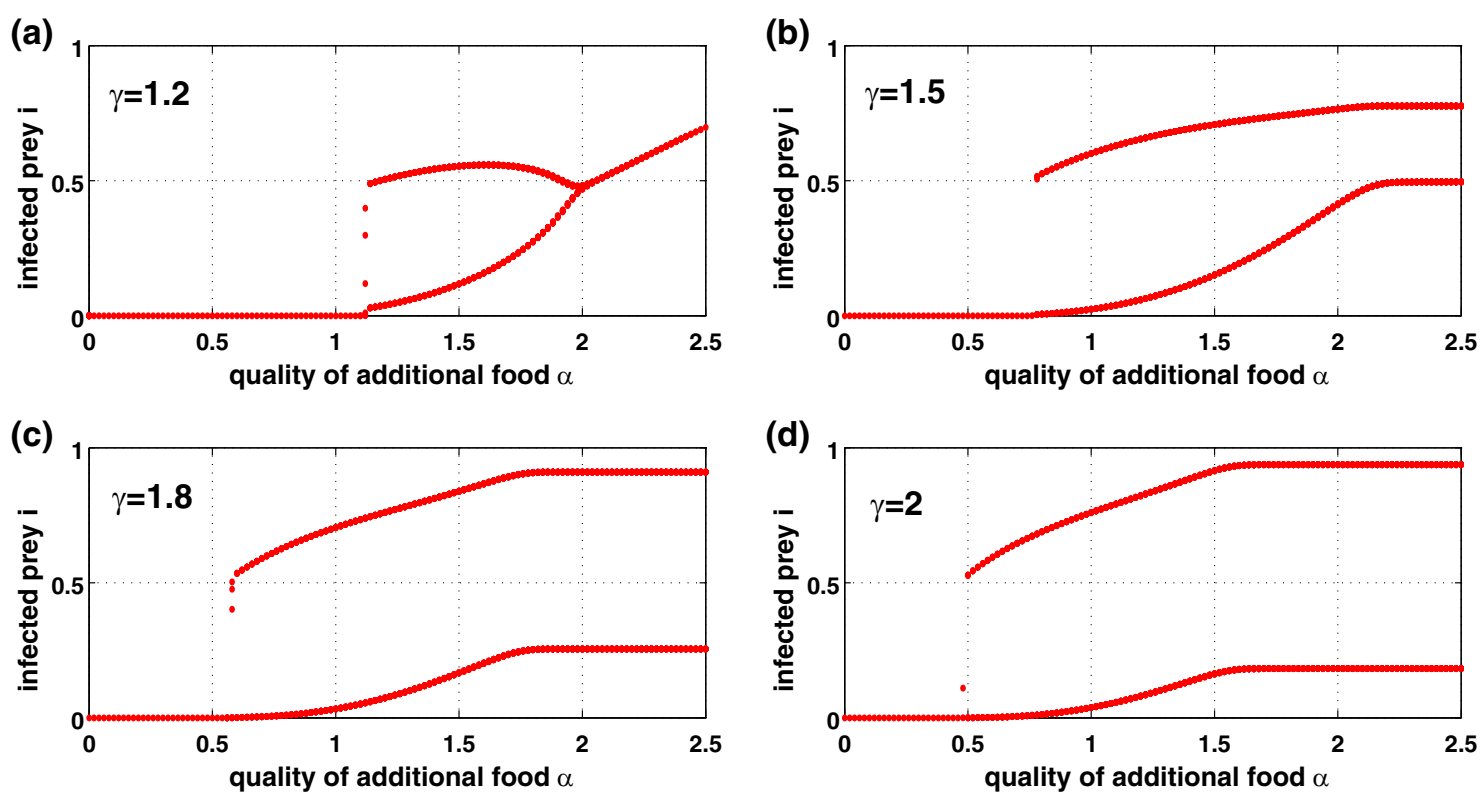

Fig. 7 Bifurcation diagram of the system (2) with respect to quality of additional food $\alpha$ for fixed quantity of additional food $\xi=0.5$ and for fixed infection rate $\mathbf{a} \gamma=1.2, \mathbf{b} \gamma=1.5$. $\mathbf{c} \gamma=1.8$ and $\mathbf{d} \gamma=2$
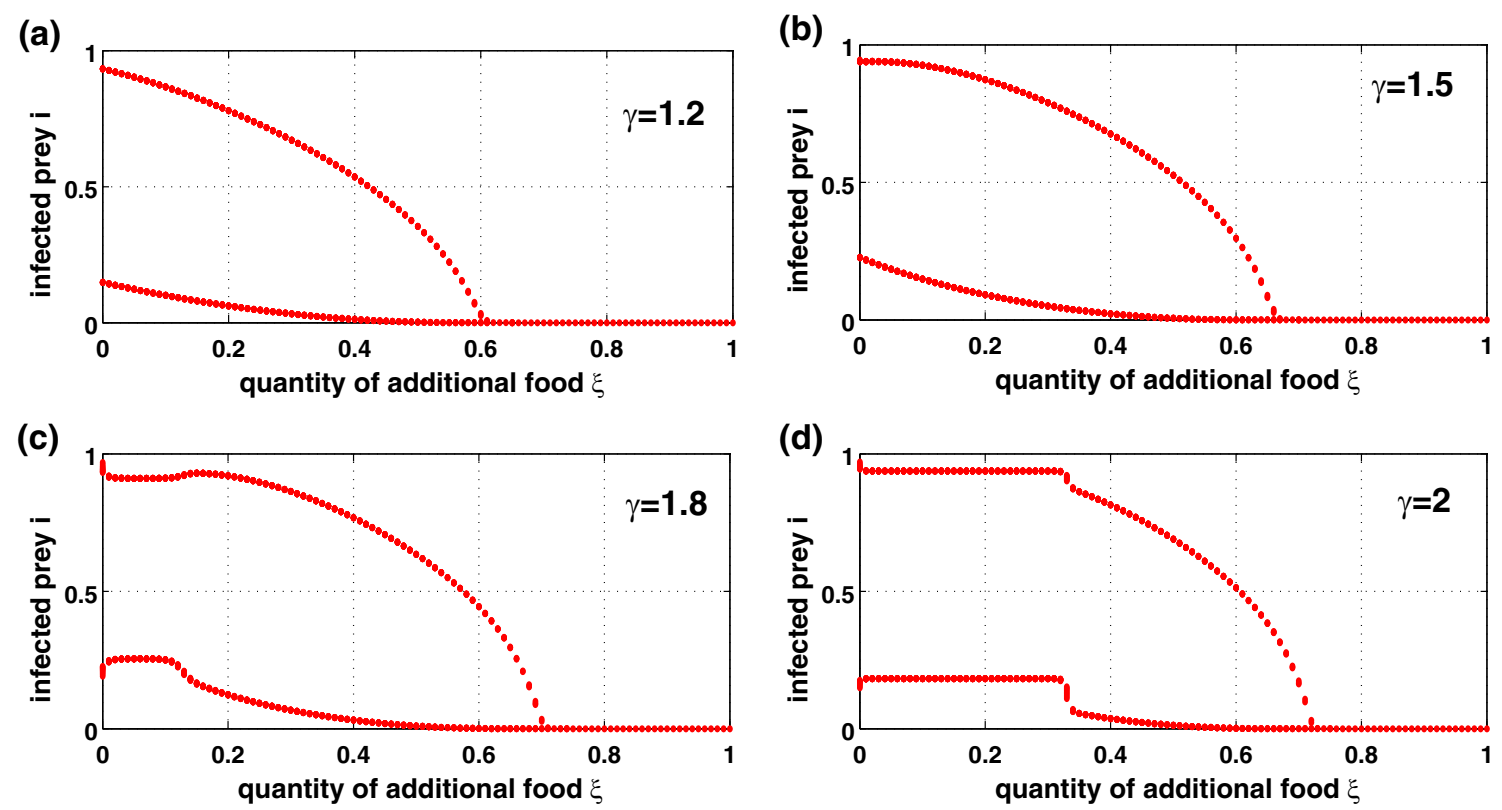

Fig. 8 Bifurcation diagram of the system (2) with respect to quantity of additional food $\xi$ for fixed quality of additional food $\alpha=0.8$ and for fixed infection rate $\mathbf{a} \gamma=1.2, \mathbf{b} \gamma=1.5$. $\mathbf{c} \gamma=1.8$ and $\mathbf{d} \gamma=2$

Figure 7 represents the bifurcation diagram of infected prey with respect to quality of additional food $(\alpha)$ for fixed infection rate $\gamma$ and fixed quantity of additional food $(\xi=0.5)$. From Fig. 7, it is clear that the system becomes disease free for $0 \leq \alpha \leq 1.12$ when $\gamma=1.2$, for $0 \leq \alpha \leq 0.78$ when $\gamma=1.5$, for $0 \leq \alpha \leq 0.57$ when $\gamma=1.8$ and within $0 \leq \alpha \leq 0.48$ when $\gamma=2$. Therefore, infection of the system can be controlled providing high quality of additional food. We also plot bifurcation diagrams of infected prey with respect to quantity of additional food $\xi$ for fixed quality of additional food $\alpha=0.8$ and fixed infection rate $\gamma$ in Fig. 8. From Fig. 8 , it is evident that the system is disease free for $0.56 \leq \xi \leq 1$ when $\gamma=1.2$, for $0.68 \leq \xi \leq 1$ when $\gamma=1.5$, for $0.72 \leq \xi \leq 1$ when $\gamma=1.8$ and for $0.74 \leq \xi \leq 1$ when $\gamma=2$. Therefore, for higher infection rate $(\gamma>0.94)$, high quality of additional food is required to make the system disease free. We also present the effects of additional food on infected prey population with respect 

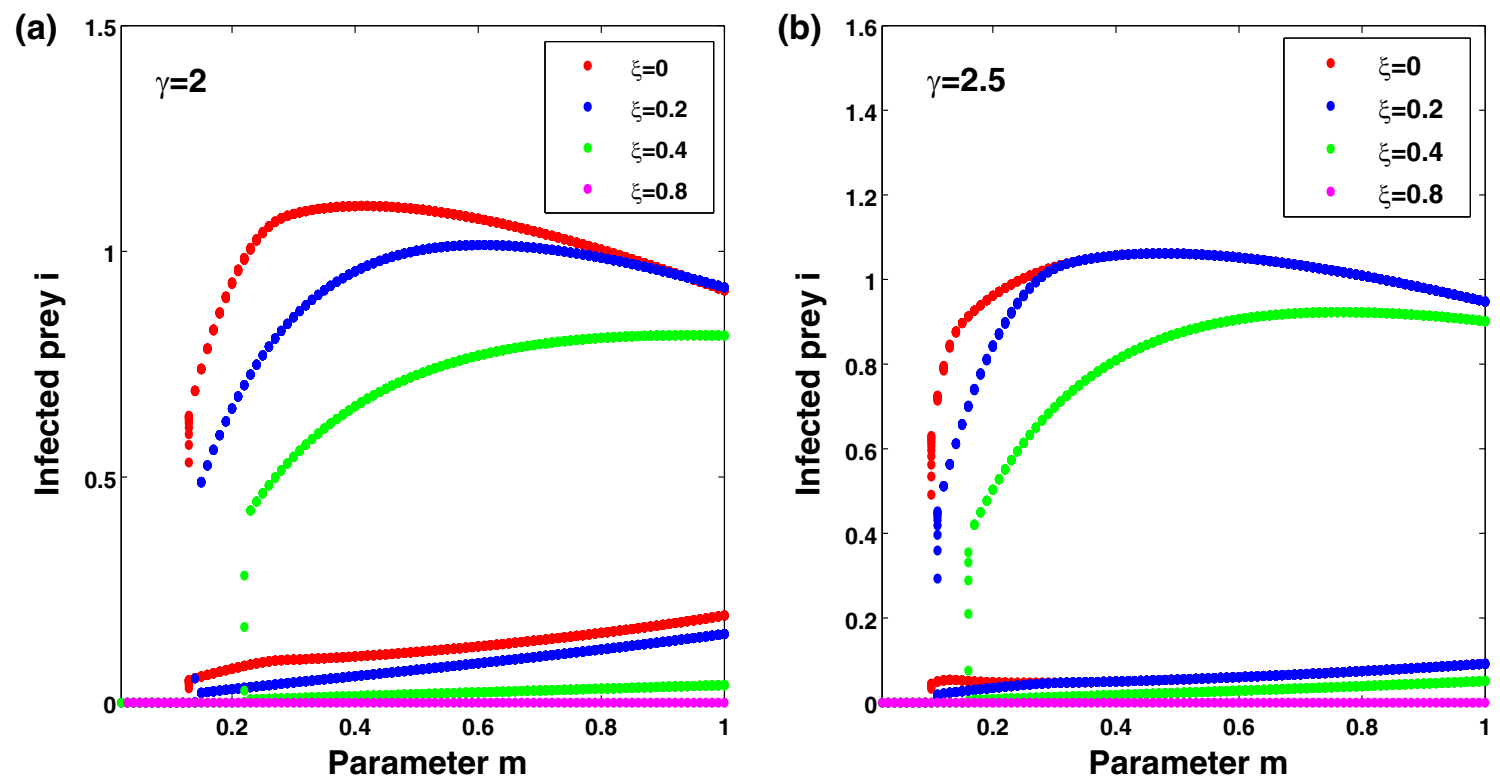

Fig. 9 Bifurcation diagram of the system (2) with respect to the parameter $m$ for different quantity of additional food $\xi$ with fixed quality of additional food $\alpha=0.8$ and fixed infection rate $\mathbf{a} \gamma=2, \mathbf{b} \gamma=2.5$

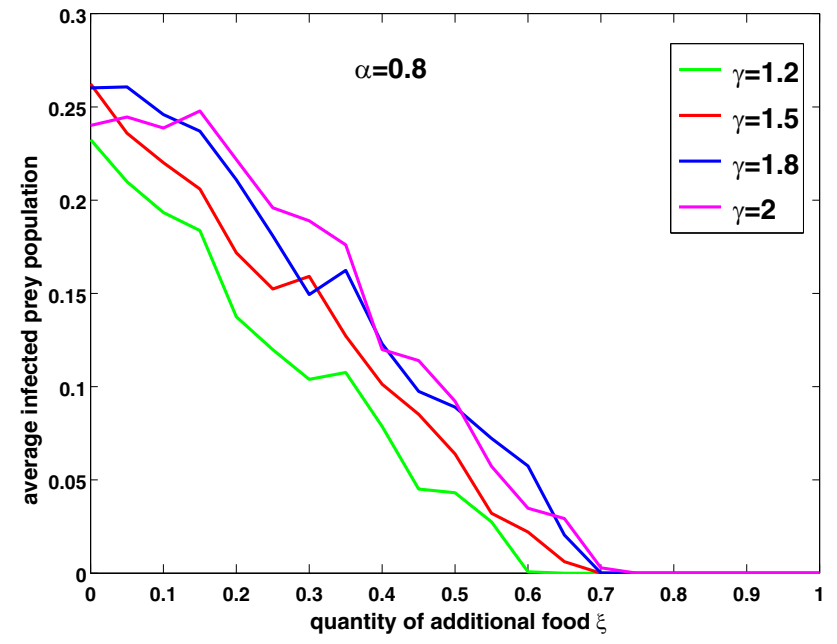

Fig. 10 Results of average infected prey population with respect to quantity of additional food for different infection rates

to the parameter $m$ (inverse of latent period) in Fig. 9 for fixed infection rate. From Fig. 9, we observe that infected prey individuals exist for higher $m$, but prey population becomes infection free for suitable supply of additional food to predator. The average infected prey population is also found and plotted in Fig. 10. It is clear that average growth of infected prey decreases for increase rate of quantity of additional food and ultimately infected preys extinct. Therefore, our investigation establish that disease control is possible supplying high quality and high quantity of additional food.

We now plot infected and uninfected regions in the $\gamma \alpha$ and $\gamma \xi$ planes respectively considering the parameters values of Table 2 and the regions are presented in Fig. 11. In Fig. 11a, the $\gamma \alpha$ plane is divided into infected and uninfected regions for $0.94 \leq \gamma \leq 3.5$ and $0 \leq \alpha \leq 2$ with fixed quantity of additional food $\xi=0.5$. In Fig. $11 \mathrm{~b}$, we plot $\gamma \xi$ plane for $0.94 \leq \gamma \leq 3.5$ and $0 \leq \xi \leq 1.5$ with constant quality of additional food $\alpha=0.8$. From Fig. 11a, it is clear that for higher infection rate $(\gamma>0.94)$, the disease free system exists for high quality $(0<\alpha<1)$ of additional food supply. On the other hand, for higher infection rate, the infected prey extinct from the system for higher quantity of additional food (Fig. 11b). Our above studies help to determine suitable additional food to make the system disease free.

\section{Seasonally varying contact rate}

It is well known that seasonal variations in temperature, rainfall and resource availability are ubiquitous and have strong influence on population dynamics. Since seasonality factors can influence epidemiological parameters, a major aim is to understand how environmental drivers alter the dynamics of infectious diseases. There are many diseases models incorporating seasonality (London and Yorke 1973; Yorke et al. 1979; Uziel and Stone 2012; Aron and Schwartz 1984; Stone et al. 2007; Buonomo 2011). In order to investigate the qualitative effects of seasonality, we assume the infection-rate $\gamma(t)=\gamma_{0}\left[1+\gamma_{1} \sin (2 \pi t)\right]$, where $\gamma_{0}$ is the average contact rate-constant, $\gamma_{1}$ represents the strength of the seasonal forcing between zero and unity and $t$ has units of 
(a)

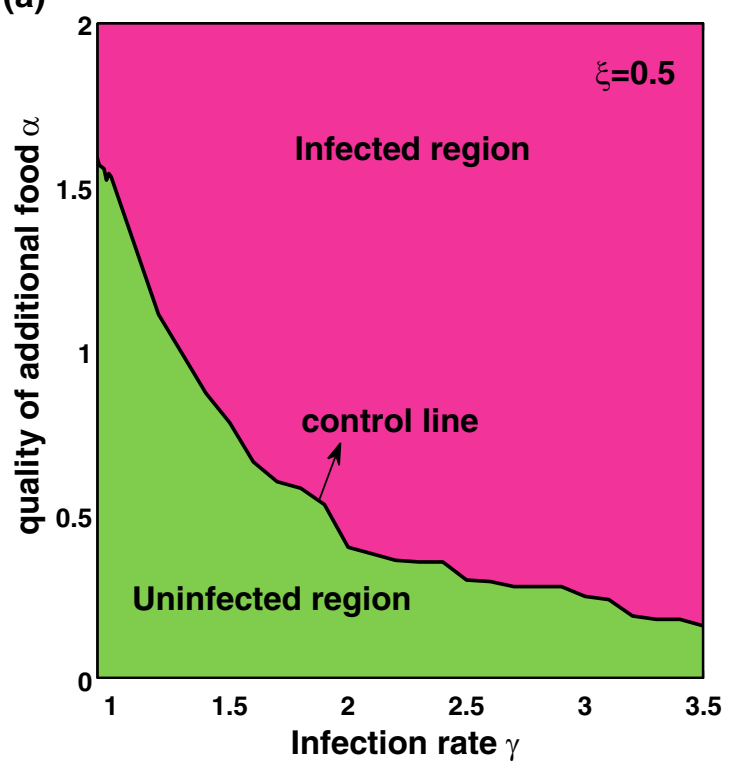

Fig. 11 Graph of $\mathbf{a} \gamma$ versus $\alpha$ for fixed $\xi=0.5$ and $\mathbf{b} \gamma$ versus $\xi$ for fixed $\alpha=0.8$. Parameters are as in Table 2. The $\gamma \alpha$ and $\gamma \xi$-planes are divided into infected and uninfected regions. The figure a depicts the

(a)

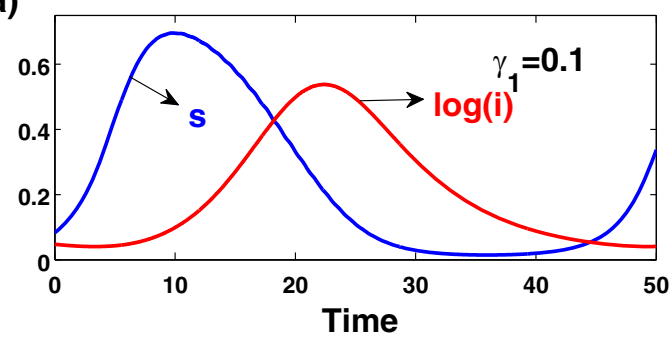

(c)

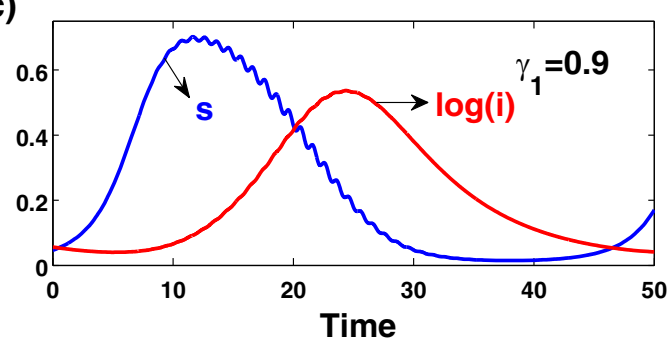

Fig. 12 Effects of seasonality on population dynamics. a Time-series simulation of infected (red line) and susceptible (blue line) prey. $\mathbf{b}$ Phase plane diagrams with the infected prey $(\log (\mathrm{i}))$ plotted as a function of susceptible preys $(s)$ for a periodic cycle. Parameters values used for $\mathbf{a}$ and $\mathbf{b}: \gamma_{0}=0.664, \gamma_{1}=0.1, \alpha=0, \xi=0$ and others

years (London and Yorke 1973; Yorke et al. 1979; Uziel and Stone 2012; Aron and Schwartz 1984; Stone et al. 2007; Buonomo 2011). Note that, for the classical epidemic model with contact rate ruled by mass action law, the sinusoidal forcing may strongly influence the long term behaviour (Buonomo 2011). Here, we show how (b)

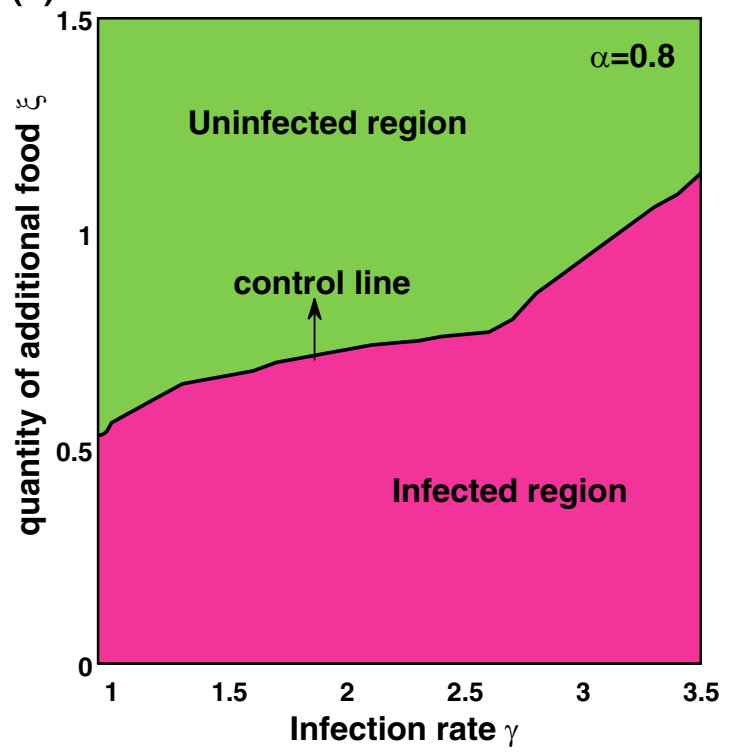

values of $\alpha$ for different infection rate $\gamma$ to make the system disease free and $\mathbf{b}$ gives the minimum values of $\xi$ for different infection rate $\gamma$

(b)

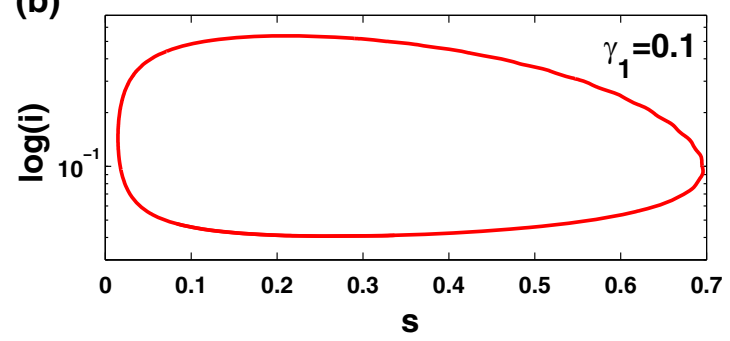

(d)

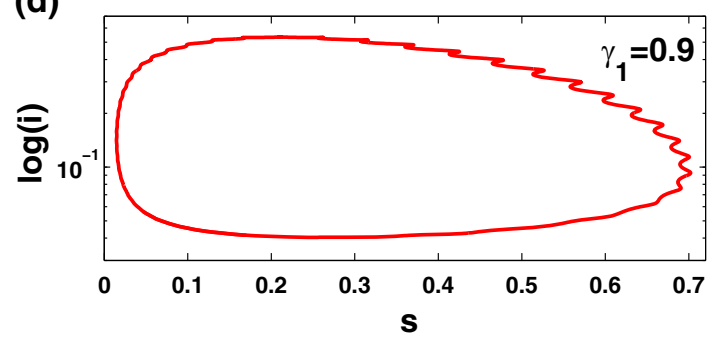

are as in Table 2. c Time-series simulation of infected prey (red line) and susceptible (blue line) prey. d Phase plane diagrams with the infected prey $(\log (i))$ plotted as a function of susceptible preys $(s)$ for a periodic cycle. Parameters values used for $\mathbf{c}$ and $\mathbf{d}: \gamma_{0}=0.664$, $\gamma_{1}=0.9, \alpha=0, \xi=0$ and others are as in Table 2

seasonality can be represented mathematically and their effects on the system (2) supplying alternative food to predator. London and Yorke (1973) demonstrated that seasonality is necessary for perpetuating a recurrent epidemic, so we will concentrate on examining variation $\gamma_{1}$ in the model (2). 
(a)

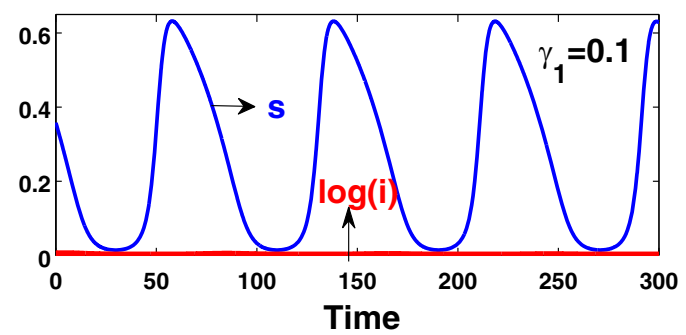

(c)

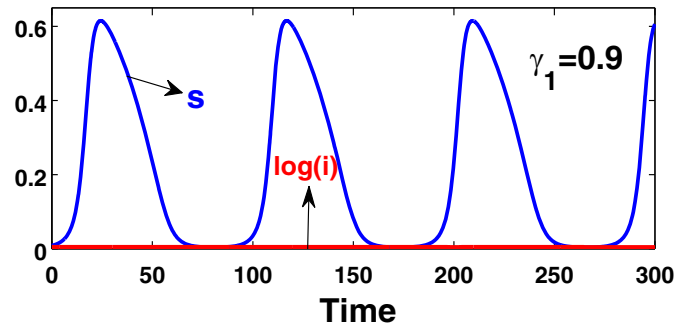

Fig. 13 Effects of seasonality on population dynamics. a Time-series simulation of infected prey (red line) and susceptible (blue line) prey. b Phase plane diagrams with the infected prey $(\log (\mathrm{i}))$ plotted as a function of susceptible preys $(s)$ for a periodic cycle. Parameters values used for $\mathbf{a}$ and $\mathbf{b}: \gamma_{0}=0.664, \gamma_{1}=0.1, \alpha=0.8, \xi=0.5$ and others are taken from Table 2. c Time-series simulation of infected

We focus on the dynamics of infected preys in presence of seasonality using the parameters values as in Table 1 . Figure 12 depicts stable periodic solutions of the model (2) using seasonal forcing. For each level of seasonal variation $\gamma_{1}$ there is a pair of figures-one plotting the logarithm of infectives versus time and other plotting the logarithm of infectives versus susceptible in absence of additional food. For $\gamma_{1}=0.1$, very weak seasonal variation of infection rate a stable limit cycle appears from the endemic equilibrium point (Fig. 12a, b). For $\gamma_{1}=0.9$, the stable limit cycle solution persists (Fig. 12c, d). From Fig. 12c, it is observed that there is very small fluctuations in the amplitude of many peaks of infected prey. The peaks mark the years where the epidemic produces a large number of cases and infection recurs in many times. An important feature of many outbreak is that alternating years of high and low incidence begin to appear. On the other hand, in presence of additional food $(\alpha=0.8, \xi=0.5)$, from Fig. 13 it is evident that the infected prey extinct from the system with weak as well as strong seasonal variation. Therefore, in low as well as high seasonal variation, an infected system can be made disease free supplying suitable additional food to predator. This behavior of the system is also clearly demonstrated through bifurcation diagram of the system.

Figure 14 is the bifurcation diagram of infected prey of the system (2) with the variation of strength of seasonal forcing $\gamma_{1}$ within $0 \leq \gamma_{1} \leq 1$ in absence of additional food. It is clear from Fig. 16 that the infected prey exists in the
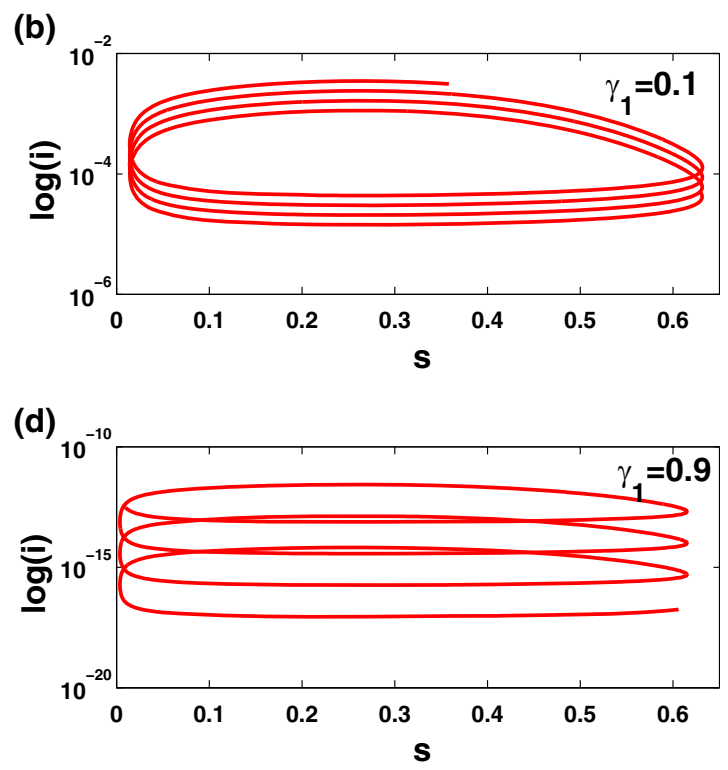

prey (red line) and susceptible (blue line) prey. d Phase plane diagrams with the infected prey $(\log (i))$ plotted as a function of susceptible preys $(s)$ for a periodic cycle. Parameters values used for $\mathbf{c}$ and $\mathbf{d}: \gamma_{0}=0.664, \gamma_{1}=0.9, \alpha=0.8, \xi=0.5$ and others are taken from Table 2

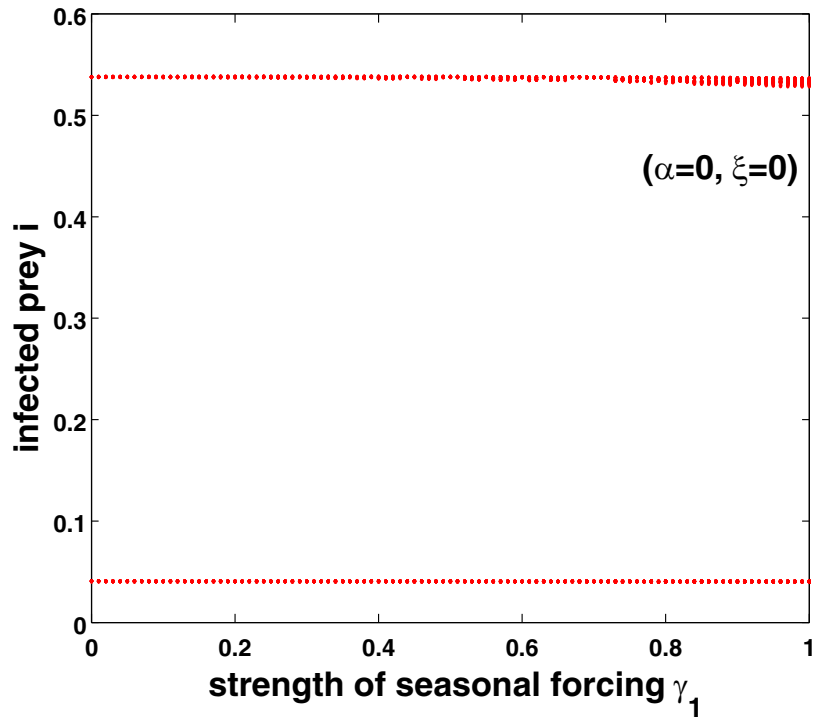

Fig. 14 Bifurcation diagram of infected prey of the system (2) with respect to strength of the seasonal forcing $\gamma_{1}$ in absence additional food i.e., for $\alpha=0$ and $\xi=0$

system with seasonal variation in absence of additional food. On the other hand, Figs. 15, 16 represent the scenarios of infected prey due to variation of seasonal parameters in presence of additional food. From Figs. 15, 16 , it is clear that suitable supply of additional food can make the system disease free in case of seasonality varying infection rate also. An important observation is that for 

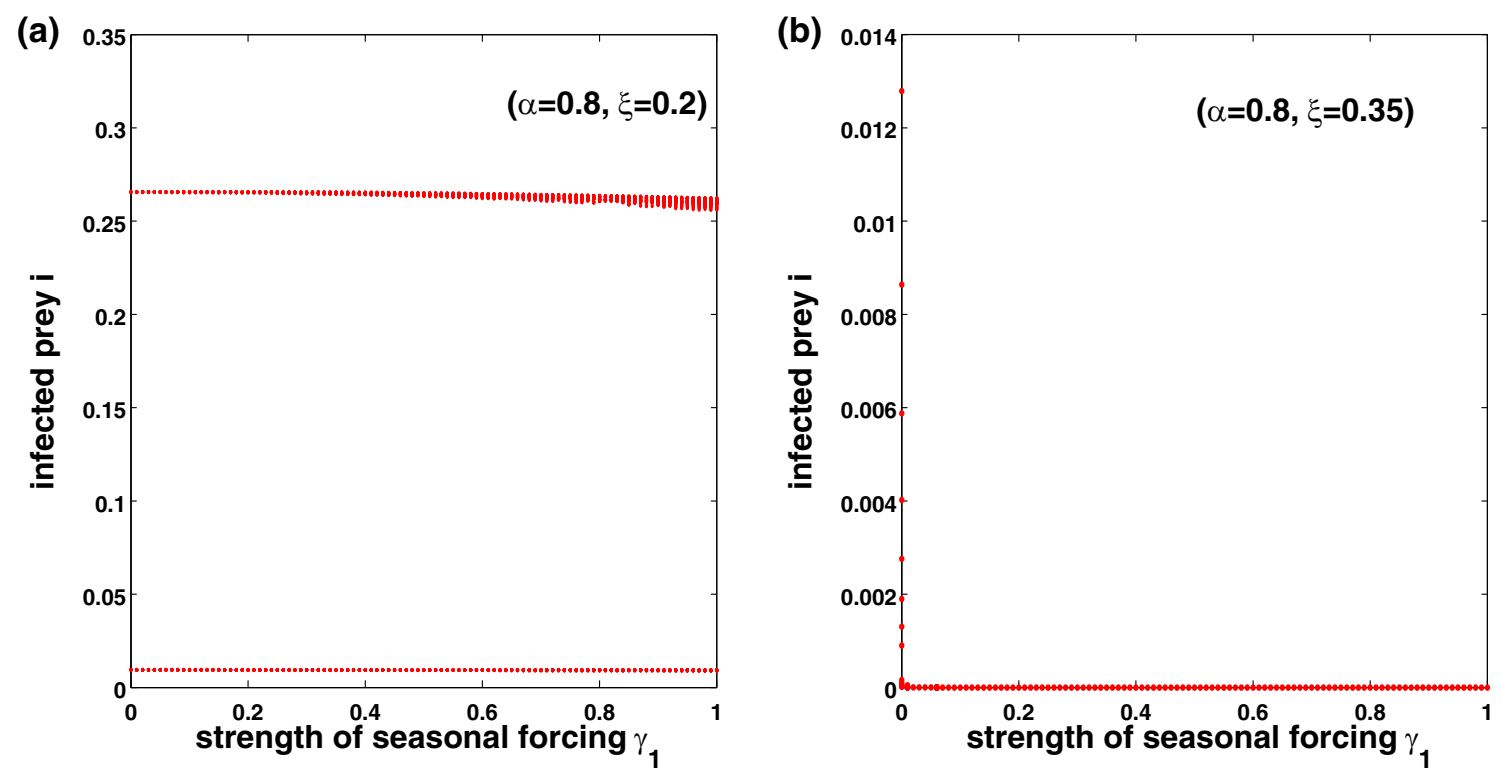

Fig. 15 Bifurcation diagram of infected prey with respect to strength of the seasonal forcing $\gamma_{1}$ in presence of high quality $(\alpha=0.8<1)$ and with quantity $\mathbf{a} \xi=0.2, \mathbf{b} \xi=0.35$ of additional food

(a)

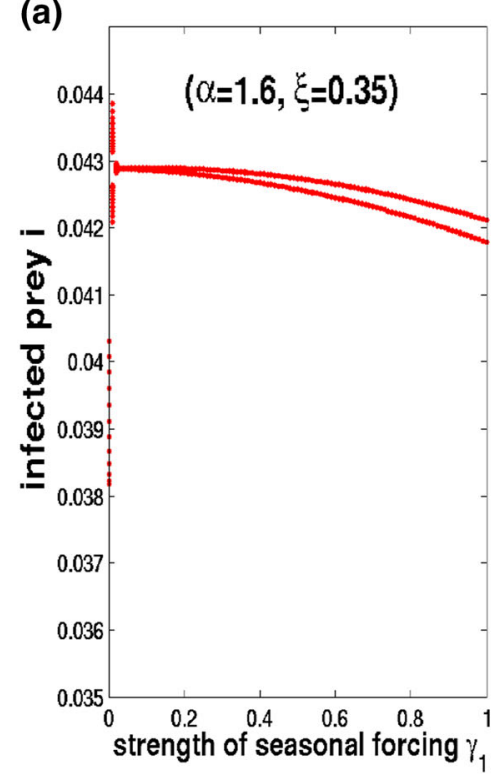

(b)

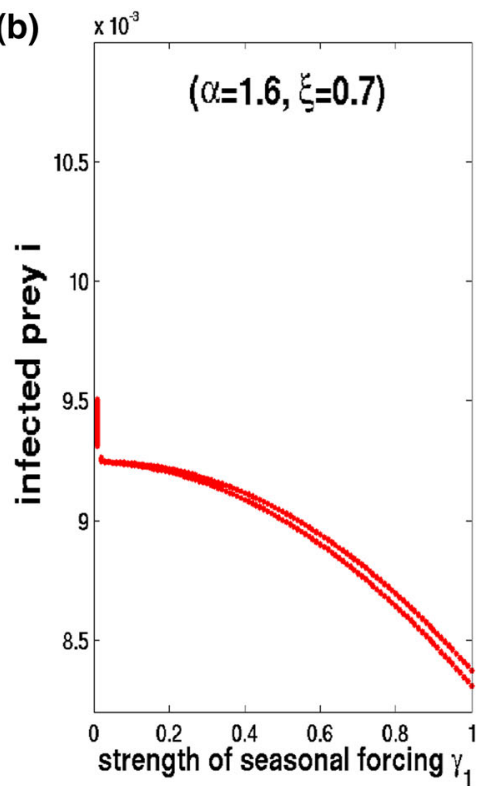

(c)

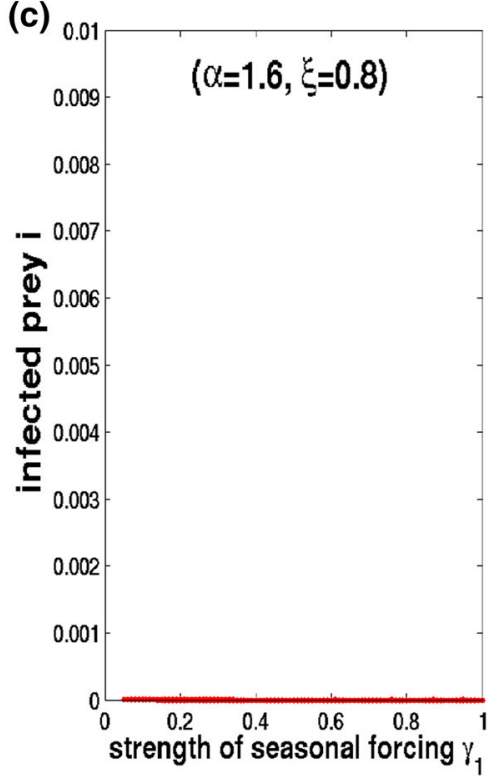

Fig. 16 Bifurcation diagram of infected prey with respect to strength of the seasonal forcing $\gamma_{1}$ in presence of low quality $(\alpha=1.6>1)$ and with quantity $\mathbf{a} \xi=0.35, \mathbf{b} \xi=0.7$ and $\mathbf{c} \xi=0.8$ of additional food

high quality $(\alpha<1)$, low quantity of additional food is sufficient to make the system disease free (Figs. 15, 16). But, for low quality $(\alpha>1)$ of additional food, high quantity is required for eradication of infected prey. So, we plot quantity of additional food as a function of quality of additional food in Fig. 17. From Fig. 17, it is clear that suitable additional food can be determined to control the diseased system. Notice in Fig. 17 that the boundaries between the infected and uninfected regions are not perfectly distinct, because there is some degree of sensitivity to small changes in parameter values resulting in sharp transitions between these outcomes.

\section{Conclusions}

We have proposed a infected prey-predator model supplying additional food to predator. The prey population is divided into susceptible-exposed and infected classes. We 


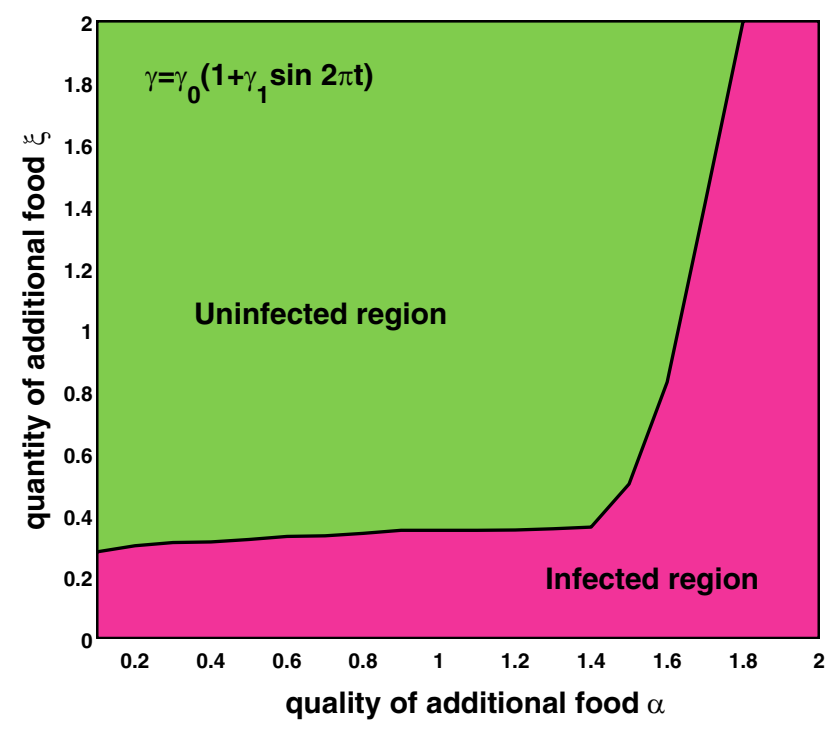

Fig. 17 Visualization of infected and uninfected regions of the system (2) in presence of seasonal variation of infection rate $\gamma=$ $\gamma_{0}\left(1+\gamma_{1} \sin (2 \pi t)\right)$ in $\alpha \xi$ plane. The figure depicts the suitable quantity $(\xi)$ of additional food for different quality $(\alpha)$ to make the system disease free

have first studied the disease free as well as predator free subsystems to observe complete picture of the full system. We obtain conditions for both local and global stability of steady state solutions of the two subsystems. We also derive the local stability criterion of the equilibrium points of the full system. We have calculated basic reproduction numbers and observed that disease free equilibrium point is locally asymptotically stable for $R_{0}^{p}<1$ and $R_{0}^{i}<1$. We also predict that the system will not be disease free if $R_{0}^{i}>1$. Bifurcation diagrams of the system are presented with respect to infection rate, quality and quantity of additional food using experimental and field data. Effects of variation of latent period is shown. The dynamics of infected prey population is also examined considering seasonal variation of the parameters.

Numerical results show that the system becomes disease free for lower infection rate $(0 \leq \gamma \leq 0.94)$ in absence of additional food (Fig. 4). This happens because infected prey is weaker than a susceptible prey and is easily captured by predator. This will reduce the number of infected prey and ultimately the system may becomes disease free in absence of additional food at certain stage for lower infection rate. But, for higher infection rate $(\gamma>0.94)$, the system can not reach disease free state without additional food (Fig. 4). Whereas for higher infection rate disease free state may be reached in presence of suitable additional food (Figs. 5, 6, 7, 8). We observe that infected prey may not be eradicated for low quality $(\alpha>1)$ of additional food for higher infection rate (Fig. 7). But, high quality of additional food $(\alpha<1)$ has the capacity of making the system disease free for higher infection rate (Fig. 7). From Fig. 7, it is clear that quality of additional food supply depends on rate of infection to make disease free system. On the other hand, quantity of additional food supply should be increased with the increase of infection rate for fixed quality to obtain a disease free system (Fig. 8). Therefore, the quality and quantity of additional food supply depends on the rate of infection to make the system disease free. Also from Fig. 10, we observe that average infected population size is declined and ultimately becomes zero in presence of suitable additional food to predator. So, we present an example of relation between quality and quantity of additional food with rate of infection in Fig. 11. From Fig. 11, it is evident that suitable supply of additional food to predator can remove disease of prey.

Here, we gain further insights through the analysis of the seasonally forced epidemic model. Seasonality is a driving force that has a major effect on the spatio-temporal dynamics of natural systems and their populations. This analysis differs from previous results for construction of disease free system. In presence of the effects of seasonality, the infected individuals may not be removed without additional food (Fig. 16), but suitable supply of additional food to predator makes a disease free system and which is clearly shown in Fig. 17. Predation may indirectly increase host abundance by reducing the negative impacts of infection. Therefore, suitable additional food supply to predator is also capable to remove disease of prey in case of seasonal dependent infection rate.

Growth rate of predator will increase with the supply of additional food and as a result predator captures the infected prey population at higher rate compare to susceptible prey. Due to this, infected prey population become very very small at certain stage and consequently infection can not spread at that stage and the system becomes disease free. Another reason behind it is that, due to predation pressure mixing of susceptible prey, exposed prey and infected prey become very high and so infected prey population increases. But in presence of additional food, rate of mixing between susceptible, exposed and infected prey decreases and as a result infected prey decreases. Notice that there is a critical infection rate above which the additional food may not be useful to make the system disease free. Sih et al. (1985) reviewed predator removal experiments and found that in 54 out of 135 systems prey population declined. Similarly Cote and Sutherland (1997) reported that predator removal reduced prey population in 3 out of 11 controlled studies. Reason behind this is that predator can help to remove parasitic infection of prey population sometimes. Therefore our theoretical results are consistent with the above experimental results. Results presented in this paper motivate to understand the role of additional food to predator as a disease controller of prey in a food chain model experimentally. 
Acknowledgments The research work of S. Poria is supported by the University Grants Commission (UGC), India [F.No-8-2/2008 (NS/ PE), dated 14th December, 2011].

\section{References}

Anderson RM, May RM (1986) The invasion, persistence and spread of infectious diseases within animal and plant communities. Philos Trans R Soc Lond B 314:533-570

Aron JL, Schwartz IB (1984) Seasonality and period-doubling bifurcations in an epidemic model. J Theor Biol 110:665-679

Buonomo B (2011) On the optimal vaccination strategies for horizontally and vertically transmitted infectious diseases. J Biol Syst 19:263-279

Butzel HM, Bolten AB (1968) The relationship of the nutritive state of the prey organism Paramecium aurelia to the growth and encystment of Didinium nasutum. J Protozool 15:256-258

Chattopadhyay J, Arino O (1999) A predator-prey model with disease in the prey. Nonlinear Anal 36:747-766

Cheney DL et al (2004) Factors affecting reproduction and mortality among baboons in the Okavango Delta, Botswana. Int J Primatol 25:401-428

Cote IM, Sutherland WJ (1997) The effectiveness of removing predators to protect bird populations. Conserv Biol 11:395-405

Diekmann O, Heesterbeek JAP (2000) Mathematical epidemiology of infectious diseases. Model building, analysis and interpretation. Wiley, Chichester

Evans SB, Mech LD, White PJ, Sargeant GA (2006) Survival of adult female Elk in yellowstone following wolf restoration. J Wildl Manag 70:1372-1378

Guin LN, Haque M, Mandal PK (2012) The spatial patterns through diffusion-driven instability in a predator-prey model. Appl Math Model 36:1825-1841

Hadeler KP, Freedman HI (1989) Predator-prey populations with parasite infection. J Math Biol 27:609-631

Haque M, Chattopadhyay J (2007) Role of transmissible disease in an infected prey-dependent predator-prey system. Math Comput Model Dyn Syst 13:163-178

Haque M, Greenhalgh D (2010) When a predator avoids infected prey: a model-based theoretical study. Math Med Biol 27:75-94

Haque M, Zhen J, Venturino E (2009) An eco-epidemiological model with standard disease incidence. Math Methods Appl Sci 32:875-898

Hastings A, Powell T (1991) Chaos in a three-species food chain. Ecology 72:896-903

Kermack WO, McKendrick AG (1927) A contributions to the mathematical theory of epidemics. Proc R Soc Edinb Sect A Math 115:700-721

Kermack WO, McKendrick AG (1932) Contributions to the mathematical theory of epidemics-II. The problem of endemicity. Proc R Soc Edinb Sect A Math 138:55-83

Kermack WO, McKendrick AG (1933) Contributions to the mathematical theory of epidemics. III-further studies of the problem of endemicity. Proc R Soc Edinb Sect A Math 141:94-122
Kooi BW, Aguiar M, Stollenwerk N (2013) Bifurcation analysis of a family of multi-strain epidemiology models. J Comput Appl Math 252:148-158

London WP, Yorke JA (1973) Recurrent outbreaks of measles, chickenpox and mumps. 1. Seasonal variation in contact rates. Am J Epidemiol 98:453-468

Sahoo B, Das B, Samanta S (2016) Dynamics of harvested-predatorprey model: role of alternative resources. Model Earth Syst Environ 2:140

Sahoo B, Poria S (2013) Disease control in a food chain model supplying alternative food. Appl Math Model 37:5653-5663

Sahoo B, Poria S (2014) Effects of supplying alternative food in a predator-prey model with harvesting. Appl Math Comput 234:150-166

Sahoo B, Poria S (2014) Diseased prey predator model with general Holling type interactions. Appl Math Comput 226:83-100

Sahoo B, Poria S (2014) Oscillatory coexistence of species in a food chain model with general Holling interactions. Differ Equ Dyn Syst 22:221-238

Sih A, Crowley P, McPeek M, Petranka j, Strohmeier k (1985) Predation, competition and prey communities: a review of field experiments. Annu Rev Ecol Syst 16:269-311

Srinivasu PDN, Prasad BSRV, Venkatesulu M (2007) Biological control through provision of additional food to predators: a theoretical study. Theo Popul Biol 72:111-120

Srinivasu PDN, Prasad BSRV (2010) Role of quantity of additional food to predators as a control in predator-prey systems with relevance to pest management and biological conservation. Bull Math Biol. doi:10.1007/s11538-010-9601-9

Steele JH, Henderson EW (1992) The role of predation in plankton models. J Plankton Res 14:157-172

Stone L, Olinky R, Huppert A (2007) Seasonal dynamics of recurrent epidemics. Nature 446:533-536

Taylor N, Walters C (2010) Estimation of bioenergetics parameters for a stunted Northern Pikeminnow population of South Central British Columbia. Open Fish Sci J 3:110-121

Tomé T, de Oliveira MJ (2011) Susceptible-infected-recovered and susceptible-exposed-infected models. J Phys A Math 4:095005

Uziel A, Stone L (2012) Determinants of periodicity in seasonally driven epidemics. J Theor Biol 305:88-95

Van den Driessche P, Watmough J (2002) Reproduction numbers and sub-threshold endemic equilibria for compartmental models of disease transmission. Math Biosci 180:29-48

Venturino E (2010) Ecoepidemic models with disease incubation and selective hunting. J Comput Appl Math 234:2883-2901

Wei H, Jiang Y, Song X, Su GH, Qiu SZ (2009) Global attractivity and permanence of a SVEIR epidemic model with pulse vaccination and time delay. J Comput Appl Math 229:302-312

Yorke JA, Nathanson N, Pianigiani G, Martin J (1979) Seasonality and the requirements for perpetuation and eradication of viruses in populations. Am J Epidemiol 109:103-123

Zhou X, Cui J (2011) Analysis of stability and bifurcation for an SEIV epidemic model with vaccination and nonlinear incidence rate. Nonlinear Dyn 63:639-653 